\title{
An Integrated Aerothermoelastic Analysis Framework for Predicting the Response of Composite Panels
}

\author{
Daning Huang*, Peretz P. Friedmann ${ }^{\dagger}$ \\ Department of Aerospace Engineering \\ The University of Michigan, Ann Arbor, MI, 48109
}

\begin{abstract}
This study describes the development of an integrated aerothermoelastic computational framework. The framework consists of a Navier-Stokes aerodynamic solver based on the Stanford University multiblock (SUmb) code, a finite element structural solver for moderate deflection of composite doubly-curved shallow shell with thermal stress, and a finite element thermal solver for heat transfer in composite shallow shells with nonlinear material properties. The solvers are coupled using a partitioned scheme. An analytical approach is developed to determine the time accuracy and the so-called energy accuracy of a loosely-coupled scheme. The energy accuracy is connected to the time accuracy of damping of the predicted response, and thus connected to the accuracy of predicted critical flutter point. The aeroelastic behaviors of $2 \mathrm{D}$ and 3D panels are investigated using the computational framework. The 3D effect and Reynolds number is found to have significant influence on the critical flutter parameter, and limit cycle amplitude.
\end{abstract}

\section{List of Symbols}

Latin Symbols

$\begin{array}{ll}a_{\infty} & \text { Freestream speed of sound } \\ \mathbf{A} & \text { Extensional stiffness matrix for composite shell } \\ A_{1}, A_{2} & \text { Metrics in orthogonal curvilinear coordinate system } \\ a, b & \text { Panel dimensions } \\ a_{i} & \text { Acceleration at } i \text { th time step } \\ \mathbf{B} & \text { Extension-bending stiffness matrix for composite shell } \\ {\left[C_{T}\right]} & \text { Element heat capacity matrix } \\ {[\bar{C}]} & \text { Total damping matrix } \\ {\left[\bar{C}_{T}\right]} & \text { Total heat capacity matrix } \\ c & \text { Specific heat capacity of solid material } \\ \mathbf{D} & \text { Bending stiffness matrix for composite shell } \\ D_{x x} & \text { Bending stiffness of a panel in } x \text { direction } \\ d & \text { Structural displacements } \\ d_{i} & \text { Displacement at } i \text { th time step } \\ \mathrm{d} V & \text { Differential volume } \\ \tilde{d}_{i} & \text { Modified displacement at } i \text { th time step in Newmark- } \beta \text { scheme } \\ \Delta d & \text { Iterative increment for Newton-Raphson method } \\ E_{1}, E_{2} & \text { Young's moduli } \\ E_{t o t} & \text { The sum of kinetic energy and strain energy } \\ F & \text { Convective flux in NS equation } \\ \{F\},\left\{F_{A}\right\},\left\{F_{T}\right\} & \text { Loading vector on an element } \\ \left\{F_{c}\right\} & \text { Vector used to apply BC to loading vector in Eq. (50) } \\ \{\bar{F}\},\{\tilde{F}\} & \text { Total/Modified loading vector } \\ f & \text { Lagrangian 1D shape functions } \\ G & \text { Diffusive flux and source terms in NS equation } \\ \mathbf{G} & \text { Stiffness matrices in lamina constitutive relation } \\ H & \text { Altitude } \\ h & \text { Shell thickness } \\ & \\ & \end{array}$

${ }^{*}$ Ph.D. Candidate, Student Member AIAA.

${ }^{\dagger}$ François-Xavier Bagnoud Professor of Aerospace Engineering, Fellow AIAA. 


\begin{tabular}{|c|c|}
\hline$h_{c}$ & Heat transfer coefficient \\
\hline$h_{i}, h_{i+1}$ & Lower and upper height of the $i$ th lamina \\
\hline I & Unit inertia matrix in Eq. (45) \\
\hline$I_{\alpha}$ & Typical section moment of inertia \\
\hline$\left[I_{c}\right]$ & Matrix used to apply BC to stiffness matrix in Eq. (50) \\
\hline$J$ & Jacobian \\
\hline$K_{1}, K_{2}$ & Constants in mesh time-averaging method \\
\hline$K_{f}, K_{g}$ & Number of intermediate meshes used to compute $F$ and $G$ \\
\hline$K_{h}, K_{\alpha}$ & Spring constant for plunging and torsion \\
\hline$[K],\left[K_{L 1}\right],\left[K_{L 2}\right],\left[K_{N}\right]$ & Stiffness matrix of an element, its linear and nonlinear components \\
\hline$\left[K_{b}\right]$ & Matrix used to apply BC to thermal conductivity matrix \\
\hline$\left[K_{T}\right]$ & Element thermal conductivity matrix \\
\hline$[\bar{K}],[\tilde{K}]$ & Total/Modified stiffness matrix \\
\hline$\left[\bar{K}_{T}\right]$ & Total thermal conductivity matrix \\
\hline$K E$ & Kinetic energy \\
\hline$k=\frac{\omega b}{U_{\infty}}$ & Reduced frequency \\
\hline$k_{h} \infty_{\infty}$ & Thermal conductivity \\
\hline$\left[k_{\text {lam }}\right]$ & Thermal conductivity tensor in reference coordinate system \\
\hline$L$ & Lift of typical section \\
\hline$L_{0}, L_{1}, L_{2}, L_{3}, L_{w}, L_{w 1}, L_{w 2}$ & Matrices that transform displacements into strains in Eq. (38) \\
\hline$L_{4}$ & Matrix that transforms discrete temperatures into continuous temperature in Eq. (80) \\
\hline$L_{5}$ & Matrix that transforms discrete temperatures into temperature gradient in Eq. (82) \\
\hline$l_{1}, l_{2}, l_{3}, l_{h}$ & Dimensions of CFD mesh \\
\hline $\mathbf{M}, M_{x}, M_{y}, M_{x y}$ & Internal moments \\
\hline $\mathbf{M}_{T}$ & Moments due to thermal effect \\
\hline$M$ & Mach number \\
\hline$M_{e a}$ & Moment about elastic axis \\
\hline$m$ & Mass of typical section \\
\hline$[M]$ & Element consistent mass matrix \\
\hline$[\bar{M}]$ & Total consistent mass matrix \\
\hline$M_{n}$ & Mach number normal to the surface of the structure \\
\hline $\mathbf{N}, N_{x}, N_{y}, N_{x y}$ & Internal in-plane forces \\
\hline $\mathbf{N}_{T}$ & In-plane forces due to thermal effect \\
\hline$N$ & Shape function \\
\hline$N_{l}$ & Number of layers in a composite shell \\
\hline$O()$ & Order of magnitude \\
\hline$p$ & Pressure \\
\hline Q & Stiffness matrices in lamina constitutive relation \\
\hline$Q$ & Magnitude of heat flux \\
\hline$\{Q\}$ & Element thermal loading vector \\
\hline$\{\bar{Q}\}$ & Total thermal loading vector \\
\hline$\left\{\bar{Q}_{b}\right\}$ & Vector used to apply BC to thermal loading vector in Eq. (94) \\
\hline$\dot{q}$ & Heat flux \\
\hline$\dot{q}_{\text {surf }}$ & Surface heat flux \\
\hline$R e$ & Reynolds number \\
\hline$R_{i}$ & Curvature in $i$ direction \\
\hline$[\bar{R}]$ & Residue \\
\hline $\mathbf{S}$ & Transverse shear stiffness matrix \\
\hline$S_{\alpha}$ & Static mass moment of typical section \\
\hline $\mathbf{T}$ & The vector of unknown temperatures in HTSH element \\
\hline $\mathbf{T}_{\mathbf{S}}, T_{x}, T_{y}$ & Internal transverse shear forces \\
\hline & Absolute temperature \\
\hline$T_{c r}=\frac{\pi^{2} h^{2}}{6 \alpha(1+\nu) a^{2}}$ & Critical temperature for thermal buckling of a plate \\
\hline$T^{0}, T^{1}, T^{\alpha}$ & Variables used in Crank-Nicolson scheme \\
\hline$T_{a m b}$ & Ambient temperature \\
\hline$T_{A W}$ & Adiabatic wall temperature \\
\hline
\end{tabular}




$\begin{array}{ll}T_{b c} & \text { Prescribed boundary temperature } \\ T_{i n i} & \text { Initial temperature } \\ T_{w} & \text { Wall temperature } \\ \{T\} & \text { Total vector of unknown temperatures } \\ \Delta T & \text { Temperature increment } \\ t & \text { Time } \\ \Delta t & \text { Time step } \\ U & \text { Total strain energy } \\ U_{\infty} & \text { Freestream velocity } \\ \{U\} & \text { The vector of displacements of all DOFs in the discretized structure } \\ \mathbf{u} & \text { The vector of all DOFs in DCS9 element } \\ u, v, w & \text { Translational displacements } \\ u_{f} & \text { Conservative variables in NS equation } \\ V & \text { Cell volume } \\ v_{i} & \text { Velocity at } i \text { th time step } \\ \tilde{v}_{i} & \text { Modified velocity at } i \text { th time step in Newmark- } \beta \text { scheme } \\ W & \text { External work } \\ w_{b} & \text { Grid velocity on fluid boundary } \\ w_{f} & \text { Grid velocity } \\ x, y, z & \text { Cartesian coordinates of the shell } \\ y^{+} & \text {Nondimensional wall distance for CFD mesh } \\ Z(x, y) & \text { Shell geometry }\end{array}$

\section{Greek Symbols}

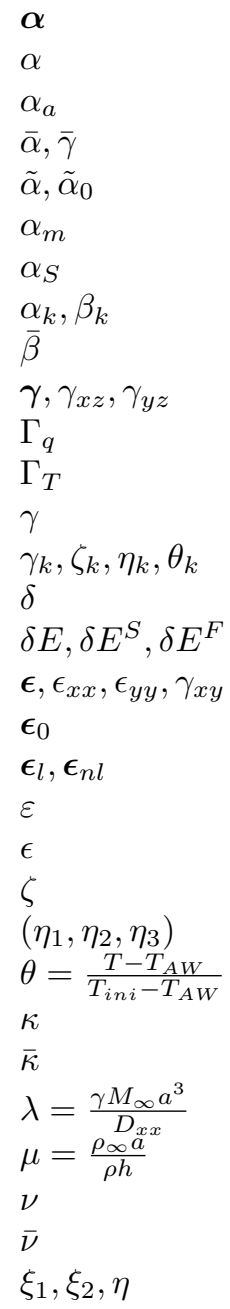

Thermal expansion coefficients in local lamination coordinate system Time dependent angle of attack Amplitude of oscillation in angle of attack

Coefficient for Crank-Nicolson scheme

Coefficients of penalty method for FE models

Mean angle of attack

Geometric angle of attack

Coefficients for mesh-averaging method

Coefficient for Newmark- $\beta$ scheme

Transverse shear strain on the middle surface

Surface where heat flux is given

Surface where temperature is prescribed

Heat capacity ratio

Coefficients for interpolation in mesh-averaging method

Boundary layer thickness at $x / a=-1, y / b=0$

Artificial energy in loosely coupled schemes

Membrane strain of the shell

Total membrane strain on the middle surface

The linear and nonlinear components of $\epsilon_{0}$

Error tolerance

Surface emissivity for thermal radiation

Damping coefficient

Coordinates on reference element

Nondimensional temperature

Shear correction factor

Weight averaged normal velocity of cell face in Eq. (5)

Nondimensional dynamic pressure

Mass ratio

Poisson's ratio

Weight averaged normal of cell face in Eq. (5)

Orthogonal curvilinear coordinate system 


\begin{tabular}{|c|c|}
\hline$\xi_{k}$ & Coefficients for interpolation \\
\hline$\Pi$ & Potential energy \\
\hline$\rho, \rho_{i}$ & Density of the material $/ i$ th lamina \\
\hline$\sigma$ & Stress tensor \\
\hline$=$ & Stefan-Boltzmann constant \\
\hline $10^{-8} \mathrm{~W} / \mathrm{m}$ & \\
\hline$\tau$ & Transverse shear stress \\
\hline$\tau$ & Thickness ratio \\
\hline$\phi$ & Phase angle \\
\hline$\chi$ & Curvature strain on the middle surface \\
\hline$\chi$ & Mesh for CFD solver \\
\hline $\bar{\chi}$ & Weight averaged mesh in mesh-averaging method \\
\hline$\tilde{\chi}$ & Reference frame for ALE formulation of NS equation \\
\hline$\chi_{f}^{(k)}$ & The $k$ th mesh for convective flux \\
\hline$\chi_{g}^{(k)}$ & The $k$ th mesh for diffusive flux \\
\hline$\Psi, \Psi_{1}$ & Coefficients for discretization error \\
\hline$\psi_{x}, \psi_{y}$ & Rotational displacements \\
\hline$\psi_{x_{i}}, \psi_{y_{i}}$ & Rotational displacements at node $i$ \\
\hline$\Omega_{T}$ & Solid domain for heat transfer \\
\hline$\tilde{\Omega}_{T}$ & The set of FE nodes that belongs to $\Omega_{T}$ \\
\hline$\omega$ & Frequency \\
\hline Acronym & \\
\hline 3PBDF & Three-Point Backward Difference Formular \\
\hline ABAQUS & A commercial finite element software \\
\hline ALE & Arbitrary Lagrangian-Eulerian \\
\hline $\mathrm{BC}$ & Boundary Condition \\
\hline CFD & Computational Fluid Dynamics \\
\hline CSD & Computational Structural Dynamics \\
\hline DCS9 & The nine-node doubly-curved shallow shell finite element \\
\hline DOF & Degree of Freedom \\
\hline DS3 & A triangular heat transfer shell elements in ABAQUS \\
\hline $\mathrm{FE}$ & Finite Element \\
\hline FSDT & First-order Shear Deformation Theory \\
\hline FSI & Fluid-Structure Interaction \\
\hline FTLT & First-order Thermal Lamination Theory \\
\hline FTSI & Fluid-Structural-Thermal Interaction \\
\hline GCL & Geometric Conservation Law \\
\hline HTSH & Heat Transfer in shallow SHell \\
\hline HYPATE & HYPersonic AeroThermoElasticity simulation environment \\
\hline $\mathrm{LCO}$ & Limit Cycle Oscillation \\
\hline NS & Navier-Stokes \\
\hline PT & Piston Theory \\
\hline RANS & Reynolds-Averaged Navier Stokes \\
\hline SITPS & Structurally Integrated TPS \\
\hline SUmb & Stanford University multi-block \\
\hline TPS & Thermal Protection System \\
\hline
\end{tabular}

Others

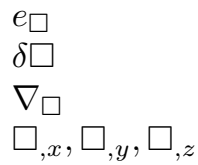

Error from

Variation operator

Del operator w.r.t. the variable

Derivative w.r.t. $x, y$, and $z$ 


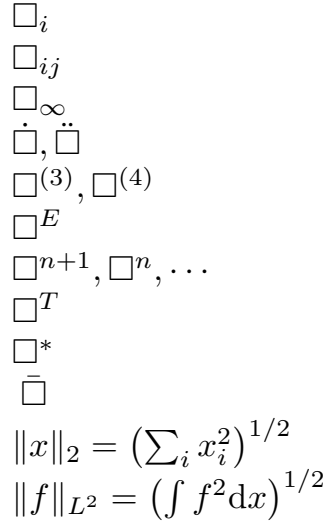

The $i$ th element in a vector

The element at $i$ th row and $j$ th column in a matrix

Quantities related to freestream

Time derivatives

Third/fourth-order time derivative

Quantities related to predictor/corrector

Quantities at time step $n+1, n, \cdots$

Transpose of a matrix

Unconstrained quantities in $\mathrm{FE}$ formulation

Amplitude

The $l^{2}$ norm

The $L^{2}$ norm

\section{Introduction}

Motivated by the interest in reusable launch vehicles for low-cost space exploration and rapid response to global military threats, hypersonic flight has been an active area of research for decades [1]. There are several technical challenges associated with hypersonic vehicles that remain unresolved. On the structural side, an important issue is the simulation of the aerothermoelastic response over extended periods of time corresponding to the vehicle trajectory. Sustained hypersonic flight causes severe aerodynamic heating and leads to degradation of material properties. The thermal stress introduced by the temperature gradient and geometrical constraints can have a major effect on structural integrity and may cause buckling, panel flutter, and control surface flutter. Therefore, accurate simulation of fluid-thermal-structural interactions (FTSI) over a substantial time period is a key ingredient for analyzing performance, stability, and reliability of hypersonic vehicles, that represents a situation where the structure, propulsion and control system are tightly coupled. The main difficulty associated with aerothermoelastic simulations is the complexity introduced by the different coupling mechanisms between and within the underlying aerothermal and aeroelastic subsystems [2].

Such difficulties are best addressed using a partitioned approach [3] with loose-coupling [4]. In a partitioned approach, the fluid, thermal, and structural responses are computed by separate solvers and coupling is achieved by exchanging boundary data at the interfaces of the domains. A loosely-coupled scheme, which exchanges information between solvers only once every time step, is computationally efficient and maintains accuracy and stability of the multi-physics solution when carefully designed [4].

The loosely-coupled partitioned scheme has been applied to aerothermoelastic analysis with subcycling technique [2]. The subcycling approach uses the disparity in characteristic times, by initiating two solvers simultaneously and advancing one solver with a multiple of the time step associated with the other solver [3]. In hypersonic aerothermoelastic problems, the characteristic time scale governing the thermal response is typically two orders of magnitude larger than that of the structural response and four orders of magnitude larger than the aerodynamic response [5]. In the multicycling scheme, three different time steps are used for the thermal, structural, and fluid solvers. The subcycling technique is applied in the coupling between fluid and structural solvers, and the coupling between thermal and aeroelastic solvers. In Ref. [2], the multicycling scheme has been numerically demonstrated to be second-order time accurate and enhances the computational efficiency of the scheme.

For the aeroelastic simulation, it is shown in Ref. [6] that the energy transfer between computational fluid dynamic (CFD) and computational structural dynamic (CSD) solvers at their interface is essential for the numerical stability of partitioned schemes. The "artificial energy", which is the sum of the work done on the structure by fluid and the work done on the fluid by structure, should be zero based on physical considerations. However, due to the staggered implementation, the artificial energy can be non-zero in partitioned schemes, thus leading to divergence. With proper combination of estimators for aerodynamic loading and structural displacement, the order of time accuracy of artificial energy and thus the stability of the numerical solution can be improved [6]. An estimator can be a predictor that extrapolates existing data to next time step, or a corrector that interpolates existing data to previous time step. In the multicycling scheme employed in [2], a predictor for aerodynamic loading is used in the aeroelastic computation. However, by using a combination of estimators, the numerical stability of the multicycling scheme may be improved.

Aeroelastic and aerothermoelastic behavior of skin panels of high speed missiles has been studied since late 1950s, as shown in a comprehensive review [7]. The aeroelastic computational framework based on Navier-Stokes (NS) CFD solver for panel flutter in subsonic and supersonic flow was first developed in Ref. [8]. Recently, a fully-coupled FTSI computational framework was used to simulate the long-time-scale behavior of skin panels in hypersonic flow [5]. 
However, these studies have two limitations. First, all simulations based on NS solvers are limited to 2D flat panels, i.e. the panel is semi-finite. Theoretical study has shown that significant differences exist in aeroelastic responses of 2D and 3D panels [9]. The panel curvature may modify aeroelastic response as well [7]. Furthermore, all CFD-based simulations focus on the response of a flat panel that is aligned to flow direction, which may not represent actual operating conditions. Furthermore, the direction of the flow will rarely coincide with that of the panel, and this effect can have considerable influence on dynamical behavior of the panel, as shown in Ref. [10]. However, none of the studies addressed the problem using a fully-coupled FTSI approach.

For hypersonic vehicles, the skin panel is combined with thermal protection system (TPS) for mitigation of the severe aerodynamic heating. Currently, there are no operational air-breather except experimental vehicles. Therefore, for aerothermoelastic studies, several generic skin panels have been conceived using TPS for rocket-based vehicles, such as Refs. [11] and [12]. These generic skin panels are based on dated TPS. In current study, a new generic skin panel based on structurally integrated TPS (SITPS) concept is employed, as shown in Fig. 1. In this example, the SITPS is composed of a sandwich structure with corrugated core, which is filled by insulation materials [13]. The outer and inner panels and the webs carry the airframe loads, and the sandwich core provides insulation. The outer panel operates in elevated temperature, while the inner panel operates in insulated environment. The SITPS is expected to be compact and has a higher structural efficiency. Since SITPS is multi-layered and may be curved due to vehicle geometry, the generic skin panel has to be modeled as a composite shell to capture its structural deformation and thermal response.

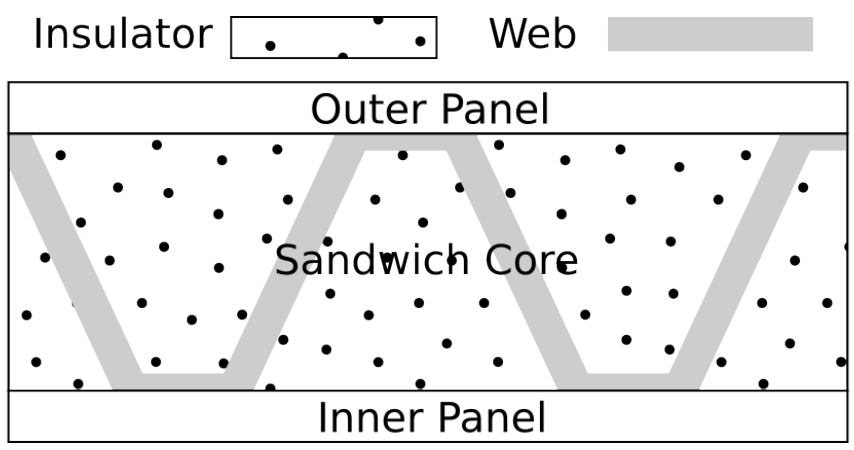

Figure 1. A typical configuration of SITPS [13].

In current study, the authors described an integrated Python-driven loosely-coupled computational framework named HYPATE, HYPersonic AeroThermoElasticity simulation environment. The framework combines an in-house developed finite element solver for structural and thermal behavior with a CFD solver based on the SUmb code [14]. An important component of the framework consists of the coupling scheme between the fluid, structure and thermal components which relies on a carefully designed loosely-coupled scheme.

The remainder of this paper is arranged as follows: Section II details the formulation of the various components of HYPATE, including the aerodynamic solver, the structural solver and the thermal solver. Section III details the analysis of the coupling schemes considered. Section IV presents the verification of the computational framework and its components. Section V presents results on the application of HYPATE to panel flutter problems. Finally, section VI provides the concluding remarks.

\section{Model Formulation}

\section{A. The Aerodynamic Solver}

The CFD solver is the modified version of the Stanford University multiblock (SUmb) solver. The original version of SUmb is a finite-volume, cell-centered multi-block solver for Euler, laminar NS, and RANS equations with steady, unsteady, and time-periodic temporal modes [14]. In current study, the Spalart-Allmaras turbulence model and perfect gas model are employed. The well-known three-point backward difference formula (3PBDF) is used for secondorder time-accurate unsteady simulation. In FTSI problems, the mesh representing fluid domain deforms according to structural displacement, which requires an appropriate implementation of an Arbitrary Lagrangian-Eulerian (ALE) formulation [15].

The central problem in ALE formulation is the implementation of the Geometric Conservation Law (GCL) condition, first proposed in Ref. [16] for structured grids and finite difference schemes. The GCL states that a flow field initialized with uniform distribution of flow states should remain unchanged during an unsteady CFD solution where 
mesh motion is present. Numerical studies have shown that violation of GCL degrades time accuracy of the CFD solution [17]. Moreover, it is pointed out in Ref. [18] that, satisfying GCL is a necessary and sufficient condition for a numerical scheme to preserve the stability of its fixed grid counterpart. There are two approaches for correct implementation of GCL in an ALE formulation. In the first approach, a source term is added to the fluid equation, so that GCL is enforced when the equation is discretized, as shown in Refs. [19] and [20]. In the second approach, a grid velocity is computed using a carefully designed interpolation scheme, as proposed in Ref. [21]. In SUmb, the second approach has been implemented [14]. However, the associated interpolation scheme for the grid velocity violates the GCL and thus it is unstable [18]. In current study, the interpolation scheme is replaced by that proposed in Ref. [21], so that the new ALE formulation in SUmb satisfies GCL, and is both stable and second-order time-accurate. Furthermore, the mesh motion in SUmb is extended from rigid body rotation and translation to arbitrary motion that includes mesh deformation.

\section{Discretization of CFD equations in ALE form}

The NS equation for dynamic mesh, i.e. the ALE formulation [15], is,

$$
\left.\frac{\partial J u_{f}}{\partial t}\right|_{\tilde{\chi}}+J \nabla \cdot\left[F\left(u_{f}\right)-w_{f} u_{f}\right]=J \nabla \cdot R\left(u_{f}\right)+J S\left(u_{f}\right)
$$

where $R$ is the diffusive flux, $S$ is the source term, and

$$
\begin{aligned}
\chi & =\chi(\tilde{\chi}, t) \\
w_{f} & =\left.\frac{\partial \chi}{\partial t}\right|_{\tilde{\chi}} \\
J & =\left|\frac{\partial \chi}{\partial \tilde{\chi}}\right|
\end{aligned}
$$

The major difference between Eq. (1) and NS equations for fixed mesh is the inclusion of mesh velocity $w_{f}$, which describes the motion of mesh $\chi$ relative to the reference frame $\tilde{\chi}$.

Using the "mesh-averaging" method in Ref. [21], Eq. (1) is discretized as,

$$
\frac{3}{2}\left(V u_{f}\right)^{n+1}-2\left(V u_{f}\right)^{n}+\frac{1}{2}\left(V u_{f}\right)^{n-1}+\Delta t F\left(u_{f}^{n+1}, \bar{\nu}, \bar{\kappa}\right)=\Delta t G\left(u_{f}^{n+1}, \bar{\chi}\right)
$$

where,

$$
\begin{aligned}
& \bar{\nu}=\sum_{k=1}^{K_{f}} \alpha_{k} \varphi\left(\chi_{f}^{(k)}\right) \\
& \bar{\kappa}=\sum_{k=1}^{K_{f}} \alpha_{k} w_{f}^{(k)} \varphi\left(\chi_{f}^{(k)}\right) \\
& \bar{\chi}=\sum_{k=1}^{K_{g}} \beta_{k} \chi_{g}^{(k)}
\end{aligned}
$$

and

$$
\begin{aligned}
& \chi_{f}^{(k)}=\zeta_{k}^{n+1} \chi^{n+1}+\zeta_{k}^{n} \chi^{n}+\zeta_{k}^{n-1} \chi^{n-1} \\
& \chi_{g}^{(k)}=\eta_{k}^{n+1} \chi^{n+1}+\eta_{k}^{n} \chi^{n}+\eta_{k}^{n-1} \chi^{n-1} \\
& w_{f}^{(k)}=\frac{\theta_{k}^{n+1} \chi^{n+1}+\theta_{k}^{n} \chi^{n}+\theta_{k}^{n-1} \chi^{n-1}}{\Delta t}
\end{aligned}
$$

Here $\varphi$ is a function that maps the mesh cell surface to its normal.

In above equations, the $k$ th mesh $\chi^{(k)}$ refers to the weighted average of meshes $\chi^{n+1}, \chi^{n}, \chi^{n-1}$, which are from current and previous time steps. The convective flux term $F$ and viscous flux and source terms $G$ in Eq. (5) are computed as the numerical fluxes on the weighted average of meshes. The terms $F$ and $G$ are calculated on different mesh sequences due to physical considerations [22]. By definition, the GCL has to be enforced in the uniform flow 
field. Since uniform flow field is steady and gradient-free, viscous flux and source terms all vanish. Therefore only convective flux is relevant to GCL and it requires special treatment.

A set of parameters [21] are used for the implementation of ALE formulation of SUmb based on mesh-averaging method,

$$
\begin{aligned}
& \zeta_{1}^{n+1}=K_{1}, \quad \zeta_{1}^{n}=K_{2}, \quad \zeta_{1}^{n-1}=0, \quad \zeta_{2}^{n+1}=K_{2}, \quad \zeta_{2}^{n}=K_{1}, \quad \zeta_{2}^{n-1}=0, \\
& \zeta_{3}^{n+1}=0, \quad \zeta_{3}^{n}=K_{1}, \quad \zeta_{3}^{n-1}=K_{2}, \quad \zeta_{4}^{n+1}=0, \quad \zeta_{4}^{n}=K_{2}, \quad \zeta_{4}^{n-1}=K_{1}, \\
& \begin{array}{lll}
\theta_{1}^{n+1}=\theta_{2}^{n+1}=1, & \theta_{1}^{n}=\theta_{2}^{n}=-1, & \theta_{1}^{n-1}=\theta_{2}^{n-1}=0 \\
\theta_{3}^{n+1}=\theta_{4}^{n+1}=0, & \theta_{3}^{n}=\theta^{n}=1, & \theta_{3}^{n-1}=\theta^{n-1}=-1
\end{array} \\
& \begin{array}{lll}
\eta_{1}^{n+1}=1, & \eta_{1}^{n}=0, & \eta_{1}^{n-1}=0 \\
\alpha_{1}=\alpha_{2}=\frac{3}{4}, & \alpha_{3}=\alpha_{4}=-\frac{1}{4}, & \beta_{1}=1
\end{array}
\end{aligned}
$$

where $K_{1}=\frac{1}{2}\left(1+\frac{1}{\sqrt{3}}\right)$, and $K_{2}=\frac{1}{2}\left(1-\frac{1}{\sqrt{3}}\right)$. The ALE scheme using parameters listed in Eq. (12) is shown to be second-order time-accurate and satisfies GCL, which means the scheme is numerically stable [21].

\section{The treatment of viscous flux}

Clarification of the treatment of the viscous flux is provided next. On viscous boundaries, no-slip condition has to be enforced to match local velocity of the wall boundary. When the boundary velocities are provided externally, such as from a structural solver, then Eq. (5) is sufficient. However, if only boundary displacements are provided, boundary velocities have to be computed by interpolation. Then the right hand side of Eq. (5) is modified to include the contribution of boundary mesh velocity,

$$
\frac{3}{2}\left(V u_{f}\right)^{n+1}-2\left(V u_{f}\right)^{n}+\frac{1}{2}\left(V u_{f}\right)^{n-1}+\Delta t F\left(u_{f}^{n+1}, \bar{\nu}, \bar{\kappa}\right)=\Delta t G\left(u_{f}^{n+1}, \bar{\chi}, \bar{w}_{b}\right)
$$

where,

$$
\begin{aligned}
\bar{w}_{b} & =\sum_{k=1}^{K_{g}} \gamma_{k} w_{b}^{(k)} \\
w_{b}^{(k)} & =\frac{\xi_{k}^{n+1} \chi^{n+1}+\xi_{k}^{n} \chi^{n}+\xi_{k}^{n-1} \chi^{n-1}}{\Delta t}
\end{aligned}
$$

Expanding $g$ as a function of $w_{b}$ at $n+1$ time step,

$$
G\left(u_{f}^{n+1}, \bar{\chi}, \bar{w}_{b}\right)=G\left(u_{f}^{n+1}, \bar{\chi}, w_{b}^{n+1}\right)+\nabla_{w} G\left(u_{f}^{n+1}, \bar{\chi}, w_{b}^{n+1}\right)\left(\bar{w}_{b}-w_{b}^{n+1}\right)+O\left(\left\|\bar{w}_{b}-w_{b}^{n+1}\right\|^{2}\right)
$$

and assuming

$$
\sum_{k=1}^{K_{g}} \gamma_{k}=1, \quad \text { so that, } \sum_{k=1}^{K_{g}} \gamma_{k} w_{b}^{n+1}=w_{b}^{n+1}
$$

yields an expression similar to Eqn. 22 in Ref. [21],

$$
w_{b}^{(k)}-w_{b}^{n+1}=\frac{1}{\Delta t}\left(\xi_{k}^{n+1}+\xi_{k}^{n}+\xi_{k}^{n-1}\right) \chi^{n+1}-\left(\xi_{k}^{n}+2 \xi_{k}^{n-1}+1\right) w_{b}^{n+1}+\frac{\Delta t}{2}\left(\xi_{k}^{n}+4 \xi_{k}^{n-1}\right) \dot{w}_{b}^{n+1}+O\left(\Delta t^{2}\right)
$$

then

$$
\begin{aligned}
\bar{w}_{b}-w_{b}^{n+1} & =\frac{1}{\Delta t} \sum_{k=1}^{K_{g}} \gamma_{k}\left(\xi_{k}^{n+1}+\xi_{k}^{n}+\xi_{k}^{n-1}\right) \chi^{n+1}-\sum_{k=1}^{K_{g}} \gamma_{k}\left(\xi_{k}^{n}+2 \xi_{k}^{n-1}+1\right) w_{b}^{n+1} \\
& +\frac{\Delta t}{2} \sum_{k=1}^{K_{g}} \gamma_{k}\left(\xi_{k}^{n}+4 \xi_{k}^{n-1}\right) \dot{w}_{b}^{n+1}+O\left(\Delta t^{2}\right)
\end{aligned}
$$

If the term containing $\left(\bar{w}_{b}-w_{b}^{n+1}\right)$ in right hand side of Eq. (16) vanishes, the diffusive flux with interpolated boundary velocities is at least second-order time-accurate compared with the one with externally provided boundary 
velocities. Then the ALE scheme in the form of Eq. (13) is second-order time-accurate. This requirement is equivalent to satisfying Eqs. (20)-(22),

$$
\begin{aligned}
\sum_{k=1}^{K_{g}} \gamma_{k}\left(\xi_{k}^{n+1}+\xi_{k}^{n}+\xi_{k}^{n-1}\right) & =0 \\
\sum_{k=1}^{K_{g}} \gamma_{k}\left(\xi_{k}^{n}+2 \xi_{k}^{n-1}+1\right) & =0 \\
\sum_{k=1}^{K_{g}} \gamma_{k}\left(\xi_{k}^{n}+4 \xi_{k}^{n-1}\right) & =0
\end{aligned}
$$

Since $K_{g}=1$, due to Eq. (17), $\gamma_{1}=1$. Then,

$$
\xi_{1}^{n+1}=\frac{3}{2}, \quad \xi_{1}^{n}=-2, \quad \xi_{1}^{n-1}=\frac{1}{2}
$$

these are exactly the coefficients of a 3PBDF formula mentioned earlier. The form Eq. (13) and coefficients Eq. (12) and Eq. (23) define a modified ALE scheme that only requires transfer of boundary displacements from external solver, while maintaining the the same stability and order of time accuracy as the original scheme based on Eq. (5). The modified form is convenient in code implementation, since only a single piece of information has to be transferred to the CFD solver at every time step.

\section{B. The Structural Solver}

The most popular finite elements (FE) suitable for shells can be classified as follows: equation-based FE [23, 24], degenerated-solid FE [25, 26] and faceted FE [27]. For composite shallow shells undergoing moderate deformation, the equation-based FE is relatively simple compared to the other FE models and at the same time it has specific advantages in terms of ease of implementation and computational efficiency. The shallow shell is suitable for modeling the skin on the surface of a hypersonic vehicle.

The doubly-curved shallow shell equations with FSDT and von Karman strain (pp. 621-626 of [28]) is used in the current study and the following assumptions are used:

1. The panel is thin, i.e. the thickness-to-span ratio of the panel is less than 1/20 [29], therefore first-order shear deformation theory (FSDT) is adequate.

2. The curved structure is shallow, i.e. the span-to-radius ratio of the panel is less than 0.5 [29], therefore shallow shell theory is applicable.

3. The panel undergoes moderate rotation with small strain thus von Karman strain is adequate for modeling the deformation.

4. No change in thickness due to external loading, i.e. total normal strain in thickness direction is neglected.

5. Under non-uniform thermal loading, the traditional composite constitutive relation with thermal strain can be used.

In HYPATE, the shell element is implemented using the shallow shell equations, which is referred to as the DCS9 (Doubly-Curved Shallow shell) element. The element has nine nodes, as shown in Fig. 2, four at corners, four at midpoints and one at the center. The numbers enclosed by circles are the nodes. Every node has five DOF's: three displacements and two rotations. Second-order Lagrangian shape functions are used to interpolate the nodal variables. 

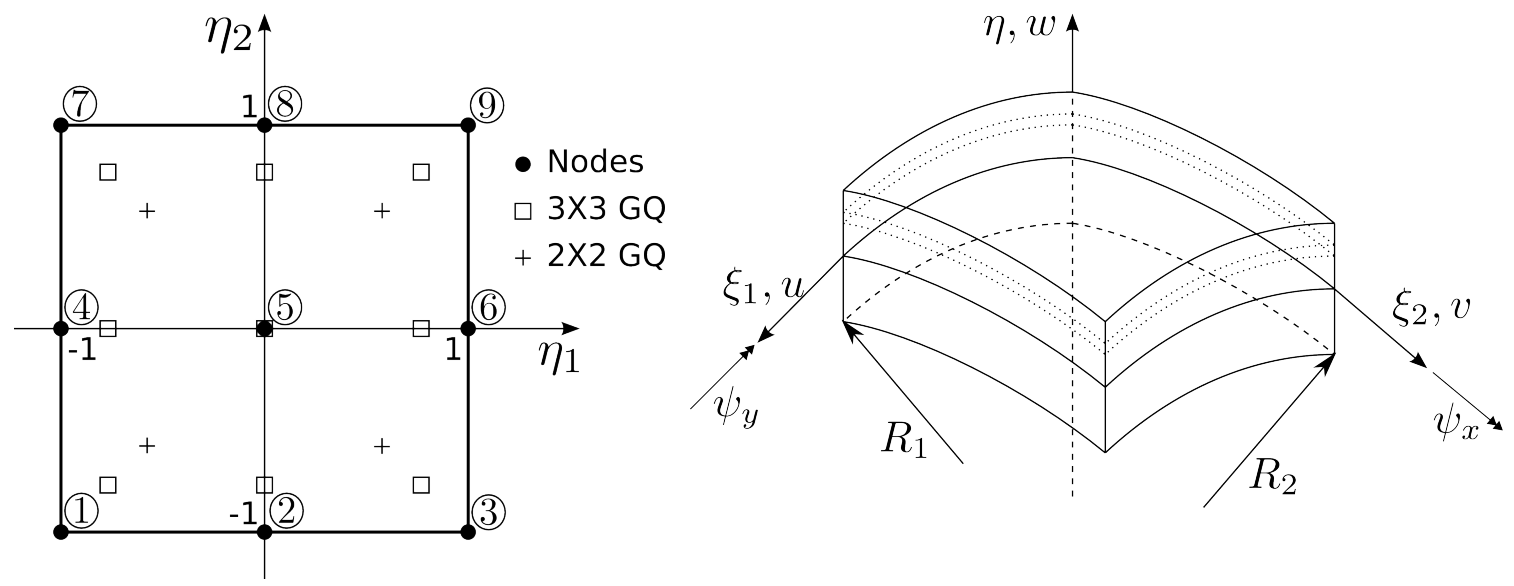

Figure 3. Local curvilinear coordinate system $\left(\xi_{1}, \xi_{2}, \zeta\right)$ in doublycurved shallow shell of laminated composites.

Figure 2. The DCS9 element in parametric coordinates.

\section{The constitutive relation}

Assuming that the shell is shallow and the curvatures are constant, the curvilinear coordinate system $\left(\xi_{1}, \xi_{2}, \zeta\right)$ of the shell is the same as global coordinate system $(x, y, z)$ and the membrane strain and the transverse shear strain by FSDT are (pp. 621-626 of [28]),

$$
\begin{aligned}
\boldsymbol{\epsilon} & =\left[\epsilon_{x x}, \epsilon_{y y}, \gamma_{x y}\right]^{T}=\boldsymbol{\epsilon}_{l}+\boldsymbol{\epsilon}_{n l}+z \boldsymbol{\chi} \\
\boldsymbol{\gamma} & =\left[\gamma_{x z}, \gamma_{y z}\right]^{T}
\end{aligned}
$$

where,

$$
\boldsymbol{\epsilon}_{l}=\left[\begin{array}{c}
u_{, x}+\frac{w}{R_{x}} \\
v_{, y}+\frac{w}{R_{y}} \\
u_{, y}+v_{, x}
\end{array}\right], \quad \boldsymbol{\epsilon}_{n l}=\left[\begin{array}{c}
\frac{1}{2} w_{, x}^{2} \\
\frac{1}{2} w_{, y}^{2} \\
w_{, x} w_{, y}^{2}
\end{array}\right], \quad \boldsymbol{\chi}=\left[\begin{array}{c}
\psi_{x, x} \\
\psi_{y, y} \\
\psi_{x, y}+\psi_{y, x}
\end{array}\right], \quad \gamma=\left[\begin{array}{c}
-\frac{u}{R_{x}}+w_{, x}+\psi_{x} \\
-\frac{v}{R_{y}}+w_{, y}+\psi_{y}
\end{array}\right]
$$

Here von Karman strain $\boldsymbol{\epsilon}_{n l}$ is used to describe moderate deflection of the shell. Furthermore, $\boldsymbol{\epsilon}_{0}=\boldsymbol{\epsilon}_{l}+\boldsymbol{\epsilon}_{n l}$ is defined as the total membrane strain.

Consider the $i$ th lamina in a laminated composite shell, which extends from $h_{i}$ to $h_{i+1}$ in the thickness direction. The constitutive relation is,

$$
\boldsymbol{\sigma}=\mathbf{Q}_{i}\left(\boldsymbol{\epsilon}+z \chi-\boldsymbol{\alpha}_{i} \Delta T\right), \quad \boldsymbol{\tau}=\mathbf{G}_{i} \boldsymbol{\gamma}
$$

where,

$$
\boldsymbol{\sigma}=\left[\begin{array}{c}
\sigma_{x x} \\
\sigma_{y y} \\
\sigma_{x y}
\end{array}\right], \quad \mathbf{Q}_{i}=\left[\begin{array}{lll}
Q_{11}^{i} & Q_{12}^{i} & Q_{13}^{i} \\
Q_{12}^{i} & Q_{22}^{i} & Q_{23}^{i} \\
Q_{13}^{i} & Q_{23}^{i} & Q_{66}^{i}
\end{array}\right], \quad \boldsymbol{\alpha}_{i}=\left[\begin{array}{c}
\alpha_{x x}^{i} \\
\alpha_{y y}^{i} \\
\alpha_{x y}^{i}
\end{array}\right]
$$

and,

$$
\boldsymbol{\tau}=\left[\begin{array}{l}
\sigma_{y z} \\
\sigma_{x z}
\end{array}\right], \quad \mathbf{G}_{i}=\left[\begin{array}{ll}
Q_{44}^{i} & Q_{45}^{i} \\
Q_{45}^{i} & Q_{55}^{i}
\end{array}\right]
$$

Assuming a known temperature distribution in the structure, integration of Eq. (27) through the thickness produces the in-plane forces $\mathbf{N}=\left[N_{x}, N_{y}, N_{x y}\right]^{T}$, moments $\mathbf{M}=\left[M_{x}, M_{y}, M_{x y}\right]^{T}$ and transverse shear forces $\mathbf{T}_{\mathbf{S}}=\left[T_{y}, T_{x}\right]^{T}$.

$$
\left(\mathbf{N}, \mathbf{M}, \mathbf{T}_{\mathbf{S}}\right)=\int_{-h / 2}^{h / 2}(\boldsymbol{\sigma}, z \boldsymbol{\sigma}, \boldsymbol{\tau}) \mathrm{d} z
$$

Since $\mathbf{Q}_{i}$ and $\boldsymbol{\alpha}_{i}$ vary from layer to layer, the integration over $\left[-\frac{h}{2}, \frac{h}{2}\right]$ has to be performed also in a piecewise manner. After the integration, one has,

$$
\begin{aligned}
{\left[\begin{array}{l}
\mathbf{N} \\
\mathbf{M}
\end{array}\right] } & =\left[\begin{array}{ll}
\mathbf{A} & \mathbf{B} \\
\mathbf{B} & \mathbf{D}
\end{array}\right]\left[\begin{array}{l}
\boldsymbol{\epsilon} \\
\chi
\end{array}\right]-\left[\begin{array}{l}
\mathbf{N}_{T} \\
\mathbf{M}_{T}
\end{array}\right] \\
\mathbf{T}_{\mathbf{S}} & =\mathbf{S} \boldsymbol{\gamma}
\end{aligned}
$$


where, $\mathbf{A}, \mathbf{B}, \mathbf{D}$ are extensional, extension-bending and bending matrices, respectively, $\mathbf{S}$ is transverse shear stiffness matrix, and $\mathbf{N}_{T}, \mathbf{M}_{T}$ are forces and moments due to thermal effect.

$$
\begin{aligned}
(\mathbf{A}, \mathbf{B}, \mathbf{D}) & =\sum_{i=1}^{N_{l}} \int_{h_{i}}^{h_{i+1}}\left(1, z, z^{2}\right) \mathbf{Q}_{i} \mathrm{~d} z \\
\mathbf{S} & =\kappa\left(\sum_{i=1}^{N_{l}} \int_{h_{i}}^{h_{i+1}} \mathbf{G}_{i} \mathrm{~d} z\right) \\
\left(\mathbf{N}_{T}, \mathbf{M}_{T}\right) & =\sum_{i=1}^{N_{l}} \int_{h_{i}}^{h_{i+1}}(1, z) \mathbf{Q}_{i} \boldsymbol{\alpha}_{i} \Delta T \mathrm{~d} z
\end{aligned}
$$

Note that a shear correction factor $\kappa$ is used in the calculation of $\mathbf{S}$. The actual transverse shear stress distribution across the thickness is non-uniform. For example, in metallic beams and plates, the distribution is parabolic. However, in FSDT, the distribution is assumed to be constant. This discrepancy is corrected by multiplying by a factor $\kappa$ to the transverse shear strain energy predicted by FSDT so that the modified transverse shear strain energy is equal to the actual transverse shear strain energy. In current study, $\kappa=\frac{5}{6}$ (pp. 135-136 of [28]).

When evaluating $\mathbf{N}_{T}, \mathbf{M}_{T}$, when the temperature is non-uniform and material properties are dependent on temperature, Simpson's rule is used to approximate the integrations in $\mathbf{A}, \mathbf{B}, \mathbf{D}, \mathbf{S}, \mathbf{N}_{T}, \mathbf{M}_{T}$. For example,

$$
\mathbf{A}=\sum_{i=1}^{N} \int_{h_{i}}^{h_{i+1}} \mathbf{Q}_{i} \mathrm{~d} z \approx \sum_{i=1}^{N}\left\{\frac{h_{i+1}-h_{i}}{6}\left[\mathbf{Q}_{i}\left(h_{i}\right)+4 \mathbf{Q}_{i}\left(\frac{h_{i}+h_{i+1}}{2}\right)+\mathbf{Q}_{i}\left(h_{i+1}\right)\right]\right\}
$$

where $\mathbf{Q}_{i}(z) \equiv \mathbf{Q}_{i}(\Delta T(z))$.

\section{Element matrices and loading vectors}

For each element, the displacement field $\overline{\mathbf{u}}=\left[u, v, w, \psi_{x}, \psi_{y}\right]^{T}$ is interpolated by the shape functions $N_{i}$ and nodal values $\mathbf{u}=\left[u_{1}, v_{1}, w_{1}, \psi_{x_{1}}, \psi_{y_{1}}, \cdots, u_{9}, v_{9}, w_{9}, \psi_{x 9}, \psi_{y_{9}}\right]^{T}$,

$$
\overline{\mathbf{u}}=L_{0} \mathbf{u}
$$

where,

$$
L_{0}=\left[\cdots \mid \begin{array}{ccccc|c}
N_{i} & 0 & 0 & 0 & 0 & \\
0 & N_{i} & 0 & 0 & 0 \\
0 & 0 & N_{i} & 0 & 0 & \\
0 & 0 & 0 & N_{i} & 0 \\
0 & 0 & 0 & 0 & N_{i}
\end{array}\right], \quad i=1, \cdots, 9
$$

and

$$
\begin{aligned}
N_{i+3 j-3} & =f_{i}(\eta) f_{j}(\xi), \quad i, j=1,2,3 \\
f_{1}(x) & =\frac{1}{2} x(x-1) \\
f_{2}(x) & =1-x^{2} \\
f_{3}(x) & =\frac{1}{2}(1+x) x
\end{aligned}
$$

The strains are,

$$
\boldsymbol{\epsilon}_{l}=L_{1} \mathbf{u}, \quad \boldsymbol{\epsilon}_{n l}=\frac{1}{2} L_{w} \mathbf{u}, \quad \chi=L_{2} \mathbf{u}, \quad \gamma=L_{3} \mathbf{u}
$$


where,

$$
\begin{aligned}
& L_{1}=\left[\cdots \mid \begin{array}{ccccc|c}
N_{i, x} & 0 & \frac{N_{i}}{R_{x}} & 0 & 0 & \\
0 & N_{i, y} & \frac{N_{i}}{R_{y}} & 0 & 0 & \cdots \\
N_{i, y} & N_{i, x} & 0 & 0 & 0 &
\end{array}\right] \\
& L_{2}=\left[\begin{array}{c|ccccc|c} 
& 0 & 0 & 0 & N_{i, x} & 0 & \\
& 0 & 0 & 0 & 0 & N_{i, y} & \cdots
\end{array}\right] \\
& L_{w}=\left[\begin{array}{c|ccccc|c}
0 & 0 & w_{, x} N_{i, x} & 0 & 0 \\
0 & 0 & w_{, y} N_{i, y} & 0 & 0 & \cdots \\
0 & 0 & w_{, y} N_{i, x}+w_{, x} N_{i, y} & 0 & 0
\end{array} \mid\right. \\
& L_{3}=\left[\begin{array}{c|ccccc|c}
0 & -\frac{N_{i}}{R_{y}} & N_{i, y} & 0 & N_{i} & \cdots \\
& -\frac{N_{i}}{R_{x}} & 0 & N_{i, x} & N_{i} & 0 &
\end{array}\right]
\end{aligned}
$$

Using constitutive relation Eq. (31), the strain energy $U$ of the shell is,

$$
U=\frac{1}{2} \int_{A}\left(\epsilon_{0}^{T} \mathbf{N}+\chi^{T} \mathbf{M}+\gamma^{T} \mathbf{T}_{\mathbf{S}}\right) \mathrm{d} A
$$

which is integrated over the middle surface of the shell.

From the variation of strain energy, the stiffness matrices and thermal loading vector is obtained,

$$
\begin{aligned}
\delta U & =\int_{A}\left(\delta \boldsymbol{\epsilon}_{0}^{T} \mathbf{A} \boldsymbol{\epsilon}+\delta \boldsymbol{\epsilon}_{0}^{T} \mathbf{B} \boldsymbol{\chi}+\delta \boldsymbol{\chi}^{T} \mathbf{B} \boldsymbol{\epsilon}_{0}+\delta \boldsymbol{\chi}^{T} \mathbf{D} \boldsymbol{\chi}+\delta \boldsymbol{\gamma}^{T} \mathbf{S} \boldsymbol{\gamma}\right) \mathrm{d} A-\int_{A}\left(\delta \boldsymbol{\epsilon}_{0}^{T} \mathbf{N}_{T}+\delta \boldsymbol{\chi}^{T} \mathbf{M}_{T}\right) \mathrm{d} A \\
& \equiv \delta \mathbf{u}^{T}\left(\left[K_{L 1}\right]+\left[K_{L 2}\right]+\left[K_{N}(\mathbf{u})\right]\right) \mathbf{u}-\left\{F_{T}\right\}
\end{aligned}
$$

where,

$$
\begin{aligned}
{\left[K_{L 1}\right] } & =\int_{A}\left(L_{1}^{T} \mathbf{A} L_{1}+L_{1}^{T} \mathbf{B} L_{2}+L_{2}^{T} \mathbf{B} L_{1}+L_{2}^{T} \mathbf{D} L_{2}\right) \mathrm{d} A \\
{\left[K_{L 2}\right] } & =\int_{A}\left(L_{3}^{T} \mathbf{S} L_{3}\right) \mathrm{d} A \\
{\left[K_{N}(\mathbf{u})\right] } & =\int_{A}\left[L_{1}^{T} \mathbf{A}\left(\frac{1}{2} L_{w}\right)+L_{w}^{T} \mathbf{A} L_{1}+L_{w}^{T} \mathbf{A}\left(\frac{1}{2} L_{w}\right)\right. \\
& \left.+L_{w}^{T} \mathbf{B} L_{2}+L_{2}^{T} \mathbf{B}\left(\frac{1}{2} L_{w}\right)\right] \mathrm{d} A \\
\left\{F_{T}\right\} & =\int_{A}\left[\left(L_{1}^{T}+L_{w}^{T}\right) \mathbf{N}_{T}+L_{2}^{T} \mathbf{M}_{T}\right] \mathrm{d} A
\end{aligned}
$$

The term $\left\{F_{T}\right\}$ is thermal loading vector. The terms $\left[K_{L 1}\right]$ and $\left[K_{L 2}\right]$ correspond to the linear part of the element stiffness matrix. Gaussian quadrature with selective reduced integration scheme is used to evaluate the integrations associated with $\left[K_{L 1}\right]$ and $\left[K_{L 2}\right]$, as shown in Fig. 2. For $\left[K_{L 1}\right]$, a set of $3 \times 3$ Gaussian quadrature points is used ("full integration"); for $\left[K_{L 2}\right]$, a set of $2 \times 2$ Gaussian quadrature points is used ("reduced integration"). This procedure avoids the "shear locking" problem in FE analysis (pp. 327-330 of [25]). The term $\left[K_{N}(\mathbf{u})\right]$ is the nonlinear part of the element stiffness matrix, which depends on and only on the nodal displacements of the element. Full integration is used for its numerical evaluation. Combining the linear and nonlinear parts yields the complete stiffness matrix,

$$
[K(\mathbf{u})]=\left[K_{L 1}\right]+\left[K_{L 2}\right]+\left[K_{N}(\mathbf{u})\right]
$$

The consistent mass matrix required for dynamic modeling is obtained from the kinetic energy $K E$,

$$
\begin{aligned}
K E & =\int_{A} \int_{-h / 2}^{h / 2} \frac{1}{2} \rho\left[\left(\dot{u}+z \dot{\psi_{x}}\right)^{2}+\left(\dot{v}+z \dot{\psi}_{y}\right)^{2}+\dot{w}^{2}\right] \mathrm{d} z \mathrm{~d} A \\
& =\frac{1}{2} \int_{A}\left[I_{1}\left(\dot{u}^{2}+\dot{v}^{2}+\dot{w}^{2}\right)+I_{2}\left(2 \dot{u} \dot{\psi}_{x}+2 \dot{v} \dot{\psi}_{y}\right)+I_{3}\left({\dot{\psi_{x}}}^{2}+{\dot{\psi_{y}}}^{2}\right)\right] \mathrm{d} A
\end{aligned}
$$


where,

$$
I_{k}=\sum_{i=1}^{N} \int_{h_{i}}^{h_{i+1}} \rho_{k} z^{k-1} \mathrm{~d} z
$$

Using the same shape functions that were used for the stiffness matrix evaluation yields the consistent mass matrix $[M]$, given by,

$$
\begin{aligned}
& {[M]=L_{0}^{T} \mathbf{I} L_{0}} \\
& \mathbf{I}=\left[\begin{array}{ccccc}
I_{1} & 0 & 0 & I_{2} & 0 \\
0 & I_{1} & 0 & 0 & I_{2} \\
0 & 0 & I_{1} & 0 & 0 \\
I_{2} & 0 & 0 & I_{3} & 0 \\
0 & I_{2} & 0 & 0 & I_{3}
\end{array}\right]
\end{aligned}
$$

Finally, distributed loading $p(x, y)$ in the $z$-direction is considered. The virtual work done by $p(x, y)$ over the element is,

$$
\delta W=\int_{A} p(x, y) \delta w \mathrm{~d} A=\left(\int_{A} \sum_{i=1}^{9} p(x, y) N_{i} \mathrm{~d} A\right) \delta w_{i}
$$

and the loading vector $\{F\}$ in the $z$-direction is,

$$
\{F\}=\int_{A} L_{0}^{T}[0,0, p(x, y), 0,0]^{T} \mathrm{~d} A
$$

\section{Solution to the nonlinear structural problem}

The stiffness and mass matrices and loading vectors of the individual elements can be assembled into the unconstrained total stiffness matrix $[\bar{K}]^{*}$, total mass matrix $[\bar{M}]$, and unconstrained total loading vector $\{\bar{F}\}^{*}$. Then penalty method is used to apply the boundary conditions (pp. 143-146 of [30]), which only modifies the stiffness matrix and the loading vector. Therefore, it is sufficient to consider the static case, the potential energy of the structure is,

$$
\Pi^{*}=\frac{1}{2}\{U\}^{T}[\bar{K}]^{*}\{U\}-\{U\}^{T}\{\bar{F}\}^{*}
$$

Suppose $N_{b}$ DOFs of the structure are constrained: the displacement of $k_{i}$ th DOFs $u_{k_{i}}$ is set to be $u_{b}^{k_{i}}, i=1,2, \cdots, N_{b}$. Then define modified potential energy as,

$$
\Pi=\frac{1}{2}\{U\}^{T}[\bar{K}]^{*}\{U\}-\{U\}^{T}\{\bar{F}\}^{*}+\frac{\tilde{\alpha}}{2} \sum_{k \in I}\left(u_{k}-u_{b}^{k}\right)^{2}, \quad I=\left\{k_{1}, k_{2}, \cdots, k_{N_{b}}\right\}
$$

where $\tilde{\alpha} \gg \max _{i, j}\left([\bar{K}]_{i j}^{*}\right)$. Then minimization of $\Pi$ leads to $u_{k}=u_{b}^{k}, k \in I$, which satisfies the constraints numerically. Taking the variation $\delta \Pi=0$ yields,

$$
\left([\bar{K}]^{*}+\left[I_{c}\right]\right)\{U\}=\{\bar{F}\}^{*}+\left\{F_{c}\right\}
$$

where,

$$
\begin{aligned}
{\left[I_{c}\right]_{i j} } & = \begin{cases}\tilde{\alpha}, & i=j \in I \\
0, & \text { else }\end{cases} \\
\left\{F_{c}\right\}_{i} & = \begin{cases}\tilde{\alpha} u_{b}^{i}, & i \in I \\
0, & \text { else }\end{cases}
\end{aligned}
$$

In practice, $\tilde{\alpha}$ is set to be,

$$
\tilde{\alpha}=\tilde{\alpha}_{0} \max _{i, j}\left([\bar{K}]_{i j}\right)
$$

where $\tilde{\alpha}_{0}$ is set to $10^{6}$ to guarantee that $\tilde{\alpha}$ is much larger than the largest entry in the stiffness matrix. Define the total stiffness matrix $[\bar{K}]$ and the total loading vector $\{\bar{F}\}$ as,

$$
\begin{aligned}
{[\bar{K}] } & =[\bar{K}]^{*}+\left[I_{c}\right] \\
\{\bar{F}\} & =\{\bar{F}\}^{*}+\left\{F_{c}\right\}
\end{aligned}
$$


Applying similar procedure to dynamic case yields the governing equations of motion given by,

$$
[\bar{M}]\{\ddot{U}\}+[\bar{C}]\{\dot{U}\}+[\bar{K}(\{U\})]\{U\}=\{\bar{F}(\{U\},\{\dot{U}\})\}
$$

Equation 52 is solved in the time domain using the Newmark- $\beta$ scheme (pp. 780-782 of [30]). Subsequently, the Newton-Raphson method is used to solve the resulting nonlinear equation.

Let $\{\ddot{U}\}=a,\{\dot{U}\}=v,\{U\}=d$ and $n$ for current time step and $n+1$ for the next time step, one has,

$$
[\bar{M}] a_{n+1}+[\bar{C}] v_{n+1}+\left[\bar{K}\left(d_{n+1}\right)\right] d_{n+1}=\left\{\bar{F}\left(d_{n+1}, v_{n+1}\right)\right\}
$$

Assuming $a_{n}, v_{n}, d_{n}$ are known, then quantities at the next time step are approximated by,

$$
\begin{aligned}
& v_{n+1}=v_{n}+(1-\bar{\gamma}) \Delta t a_{n}+\bar{\gamma} \Delta t a_{n+1} \equiv \tilde{v}_{n}+\bar{\gamma} \Delta t a_{n+1} \\
& d_{n+1}=d_{n}+\Delta t v_{n}+(1-2 \bar{\beta}) \frac{\Delta t^{2}}{2} a_{n}+\bar{\beta} \Delta t^{2} a_{n+1} \equiv \tilde{d}_{n}+\bar{\beta} \Delta t^{2} a_{n+1}
\end{aligned}
$$

and Eq. (53) becomes,

$$
\left[\tilde{K}\left(d_{n+1}\right)\right] d_{n+1}=\left\{\tilde{F}\left(d_{n+1}, v_{n+1}\right)\right\}
$$

where,

$$
\left\{\begin{array}{l}
{\left[\tilde{K}\left(d_{n+1}\right)\right]=\frac{1}{\beta \Delta t^{2}}[\bar{M}]+\frac{\bar{\gamma}}{\beta \Delta t}[\bar{C}]+\left[\bar{K}\left(d_{n+1}\right)\right]} \\
\left\{\tilde{F}\left(d_{n+1}, v_{n+1}\right)\right\}=\left\{\bar{F}\left(d_{n+1}, v_{n+1}\right)\right\}+\left(\frac{1}{\beta \Delta t^{2}}[\bar{M}]+\frac{\bar{\gamma}}{\beta \Delta t}[\bar{C}]\right) \tilde{d}_{n}-[\bar{C}] \tilde{v}_{n}
\end{array}\right.
$$

When $[\bar{M}]=0,[\bar{C}]=0$, Eq. (54) is reduced to static deformation problem. For unconditional stability, let $\bar{\gamma}=$ $\frac{1}{2}, \bar{\beta}=\frac{1}{4}$ in the calculation (pp. 780-782 of [30]).

The Newton-Raphson method is used to solve Eq. (54). In the form of a residue, the method is expressed as,

$$
[\bar{R}(d)]=[\tilde{K}(d)] d-\{\tilde{F}(d)\}=0 \rightarrow\left\{\begin{array}{l}
\left.\frac{\partial[\bar{R}(d)]}{\partial d}\right|_{d^{i}} \Delta d=-\left[\bar{R}\left(d^{i}\right)\right] \\
d^{i+1}=d^{i}+\Delta d
\end{array}\right.
$$

where $d$ is the displacement vector at $n+1$ time step, $i \geq 0$ is the iteration step and $d^{0}$ is the initial guess for the $n+1$ time step. The convergence criterion is $\|\Delta d\|_{2} /\left\|d_{i+1}\right\|_{2} \leq \varepsilon$, where $\|\bullet\|_{2}$ is $l^{2}$ norm and $\varepsilon$ is a prescribed tolerance.

Assuming $[\bar{M}]$ and $[\bar{C}]$ are constant, the Jacobian of the residue is,

$$
\frac{\partial[\bar{R}(d)]}{\partial d}=\frac{\partial\left[\bar{K}_{N}(d)\right]}{\partial d} d+[\tilde{K}(d)]-\frac{\partial\{\bar{F}(d)\}}{\partial d}-\frac{\bar{\gamma}}{\bar{\beta} \Delta t} \frac{\partial\{\bar{F}(d)\}}{\partial v}
$$

where $\left[\bar{K}_{N}(d)\right]$ is the nonlinear part of $[\bar{K}(d)]$.

To obtain the explicit forms that are suitable for programming, the first and the third terms in Eq. (57) need further elaboration. Note that $\left[\bar{K}_{N}(d)\right]$ and $\{\bar{F}(d)\}$ are linear combinations of element nonlinear stiffness matrix and loading vector, respectively. Therefore one can calculate derivatives of element nonlinear stiffness matrix and loading vector and then assemble them to obtain $\frac{\partial\left[\bar{K}_{N}(d)\right]}{\partial d} d$ and $\frac{\partial\{\bar{F}(d)\}}{\partial d}$.

For the element, only the DOF's associated with the 9 nodes, shown in Fig. 2, are involved, and one only needs to calculate $\frac{\partial\left[K_{N}(\mathbf{u})\right]}{\partial \mathbf{u}} \mathbf{u}$, for which the columns can be written explicitly as,

$$
\frac{\partial\left[K_{N}(\mathbf{u})\right]}{\partial \mathbf{u}} \mathbf{u}=\left[\frac{\partial\left[K_{N}(\mathbf{u})\right]}{\partial u_{1}} \mathbf{u}\left|\frac{\partial\left[K_{N}(\mathbf{u})\right]}{\partial v_{1}} \mathbf{u}\right| \frac{\partial\left[K_{N}(\mathbf{u})\right]}{\partial w_{1}} \mathbf{u}\left|\frac{\partial\left[K_{N}(\mathbf{u})\right]}{\partial \psi_{x_{1}}} \mathbf{u}\right| \frac{\partial\left[K_{N}(\mathbf{u})\right]}{\partial \psi_{y_{1}}} \mathbf{u} \mid \cdots\right]
$$

Nonlinear terms are only associated with $w_{i}$, therefore the derivatives of $K_{N}(\mathbf{u})$ w.r.t. $u_{i}, v_{i}, \psi_{x_{i}}, \psi_{y_{i}}$ are zero. Derivatives of $K_{N}(\mathbf{u})$ w.r.t. $w_{i}$ are,

$$
\begin{aligned}
\frac{\partial\left[K_{N}(\mathbf{u})\right]}{\partial w_{i}} \mathbf{u} & =\int_{A} \frac{\partial}{\partial w_{i}}\left[L_{1}^{T} \mathbf{A}\left(\frac{1}{2} L_{w}\right)+L_{w}^{T} \mathbf{A} L_{1}+L_{w}^{T} \mathbf{A}\left(\frac{1}{2} L_{w}\right)+L_{w}^{T} \mathbf{B} L_{2}+L_{2}^{T} \mathbf{B}\left(\frac{1}{2} L_{w}\right)\right] \mathbf{u} \mathrm{d} A \\
& =\int_{A}\left[\left(L_{1}^{T} \mathbf{A}+L_{w}^{T} \mathbf{A}+L_{2}^{T} \mathbf{B}\right) L_{w 1}+L_{w 2}^{T} \mathbf{N}\right] \mathrm{d} A
\end{aligned}
$$


where,

$$
\begin{aligned}
& L_{w 1}=\frac{\partial}{\partial w_{i}} L_{w} \mathbf{u}=\left[N_{i, x} w_{, x}, N_{i, y} w_{, y}, N_{i, y} w_{, x}+N_{i, x} w_{, y}\right]^{T} \\
& L_{w 2}=\frac{\partial}{\partial w_{i}} L_{w}=\left[\begin{array}{ccccc}
0 & 0 & N_{i, x} N_{1, x} & 0 & 0 \\
0 & 0 & N_{i, y} N_{1, y} & 0 & 0 \\
0 & 0 & N_{i, y} N_{1, x}+N_{i, x} N_{1, y} & 0 & 0
\end{array}|\cdots| \begin{array}{lllll}
0 & 0 & N_{i, x} N_{9, x} & 0 & 0 \\
0 & 0 & N_{i, y} N_{9, y} & 0 & 0 \\
0 & 0 & N_{i, y} N_{9, x}+N_{i, x} N_{9, y} & 0 & 0
\end{array}\right]
\end{aligned}
$$

The term $\frac{\partial\{\bar{F}(d)\}}{\partial d}$ is the Jacobian of the loading vector. For an element, the corresponding term is $\frac{\partial\{F(\mathbf{u})\}}{\partial \mathbf{u}}$. Similar to the stiffness matrix, only the Jacobian of element loading vector is required, as given by,

$$
\frac{\partial\{F(\mathbf{u})\}}{\partial \mathbf{u}}=\left[\frac{\partial[F(\mathbf{u})]}{\partial u_{1}}\left|\frac{\partial[F(\mathbf{u})]}{\partial v_{1}}\right| \frac{\partial[F(\mathbf{u})]}{\partial w_{1}}\left|\frac{\partial[F(\mathbf{u})]}{\partial \psi_{x_{1}}}\right| \frac{\partial[F(\mathbf{u})]}{\partial \psi_{y_{1}}} \mid \cdots\right]
$$

When the loading vector is independent of displacement, for example, $p(x, y)=$ const in Eq. (47), the Jacobian is zero. Otherwise, the Jacobian has to be evaluated explicitly.

There are two cases of particular interest, thermal loading and aerodynamic loading based on piston theory. The former one is essential to account for thermal stresses in the structure in aerothermoelastic simulations. The latter one is not required in the CFD-based computational framework, however, it will be used to verify the structural solver in section IV.

Thermal LOADING From Eq. (42), note that only derivatives w.r.t. $w_{i}$ are nonzero,

$$
\begin{aligned}
\frac{\partial\left\{F_{T}\right\}}{\partial w_{i}} & =\frac{\partial}{\partial w_{i}}\left[\int_{A}\left(L_{1}^{T}+L_{w}^{T}\right) \mathbf{N}_{T}+L_{2}^{T} \mathbf{M}_{T} \mathrm{~d} A\right] \\
& =\int_{A} \frac{\partial}{\partial w_{i}} L_{w}^{T} \mathbf{N}_{T} \mathrm{~d} A \\
& =\int_{A} L_{w 2}^{T} \mathbf{N}_{T} \mathrm{~d} A
\end{aligned}
$$

AERODYNAMIC LOADING Using full piston theory [31], assuming cavity pressure to be the same as farfield pressure $p_{\infty}$ and the flow is in $\mathrm{x}$-direction, parallel to $x-y$ plane, the loading at a point on the surface of a panel is,

$$
\frac{p}{p_{\infty}}=1-\left(1+\frac{\gamma-1}{2} M_{n}\right)^{\frac{2 \gamma}{\gamma-1}}
$$

where $M_{n}=\frac{w, t}{a_{\infty}}+M_{\infty}\left(w_{, x}+Z_{, x}\right)$.

Using Eq. (47), the aerodynamic loading vector is,

$$
\left\{F_{A}\right\}=\int_{A} L_{0}^{T}[0,0, p, 0,0]^{T} \mathrm{~d} A
$$

Again only the derivatives w.r.t. $w_{i}$ are nonzero, which is,

$$
\begin{aligned}
\frac{\partial p}{\partial w_{i}} & =-\gamma p_{\infty}\left(1+\frac{\gamma-1}{2} M_{n}\right)^{\frac{\gamma+1}{\gamma-1}}\left(\frac{\bar{\gamma}}{\bar{\beta} \Delta t a_{\infty}} N_{i}+M_{\infty} N_{i, x}\right) \\
\frac{\partial\left\{F_{A}\right\}}{\partial w_{i}} & =\int_{A} L_{0}^{T}\left[0,0, \frac{\partial p}{\partial w_{i}}, 0,0\right]^{T} \mathrm{~d} A
\end{aligned}
$$

\section{The Thermal Solver}

Several methods have been developed to simplify the FE modeling of heat transfer in composite shells [32]. These methods include first-order thermal lamination theory [33, 34], layer-wise thermal lamination theory (LTLT) [35, 36], and method of homogenization $[13,37]$. The FE based on thermal lamination theory are shell elements that use ad hoc assumptions for the temperature distribution to simplify the heat transfer problem. The method of homogenization replaces the anisotropic thermal properties of the composite shell with effective homogeneous thermal properties, so as to avoid modeling heat conduction layer by layer. To accurately capture the through-thickness temperature distribution and minimize computational cost, the LTLT method is employed in current study. 
In the LTLT based shell element, the nodes are distributed over the shell middle surface. The temperatures $T_{i}$ at interfaces between the layers are unknowns at the node, as shown in Fig. 4. The through-the-thickness temperature distribution is approximated by piecewise linear interpolation for the unknowns. In Fig. 4, the gray dashed line is the middle surface of the shell.

In HYPATE, the LTLT based shell element is implemented using a solid element, which is referred to as the HTSH (Heat Transfer in shallow SHell) element. As illustrated in Fig. 5, the HTSH element is an 8-noded element with first-order Lagrangian shape function. The nodes are labelled by numbers enclosed by circles. Every node has one unknown, namely the temperature. A series of cells are stacked in the thickness direction, each representing one layer. In this way, the through-the-thickness temperature distribution is approximated by piecewise linear interpolation, as required by the LTLT based shell element.

For the formulation of the HTSH element, following assumptions are made:

1. The panel is thin, i.e. the thickness-to-span ratio of the panel is less than $1 / 20$ [29].

2. The curved structure is shallow, i.e. the span-to-radius ratio of the panel is less than 0.5 [29].

3. The panel remains shallow when it undergoes moderate deflection.

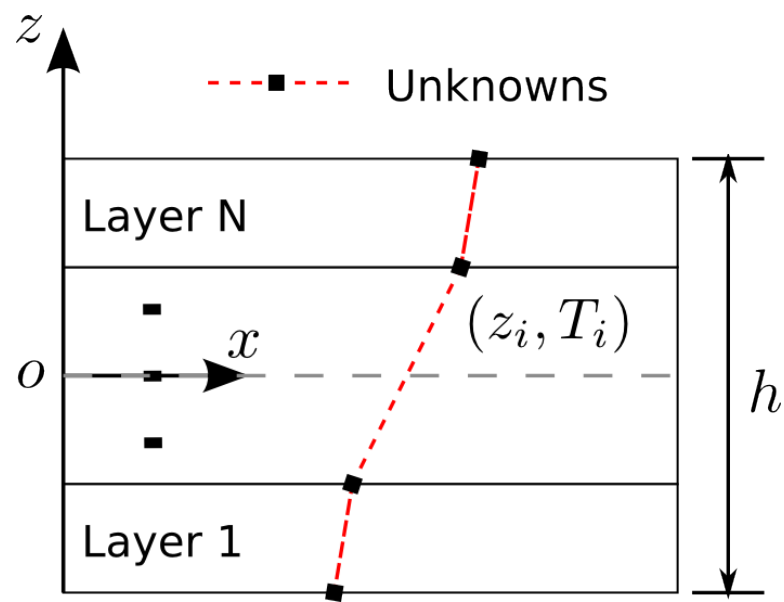

Figure 4. The LTLT based shell element using piecewise linear interpolation.

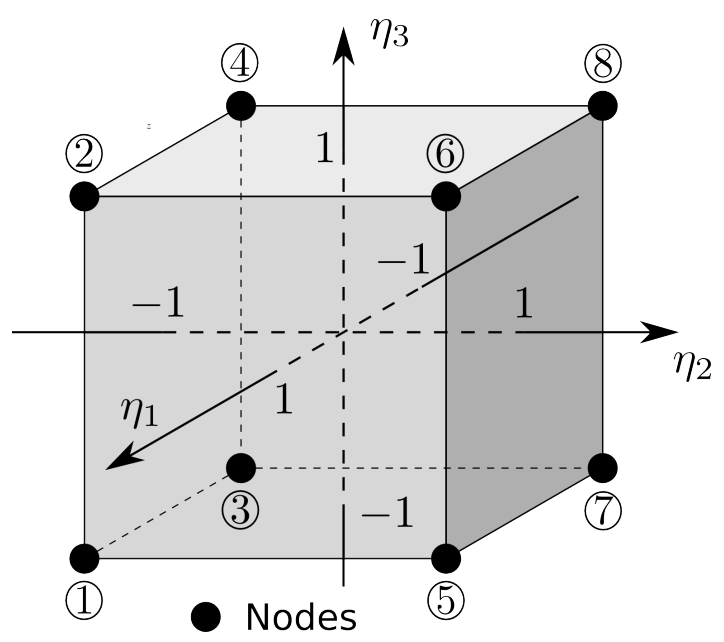

Figure 5. The HTSH element.

\section{Heat transfer equation in shallow shells}

The heat transfer equation is [38],

$$
\begin{array}{ccc}
(\rho c) \dot{T}-\nabla \cdot\left(\left[k_{\text {lam }}\right] \cdot \nabla T\right)=0 & \text { in } & \Omega_{T} \\
-\left[k_{\text {lam }}\right] \cdot \nabla T=\dot{q}_{\text {surf }} & \text { in } & \Gamma_{q} \\
T=T_{b c} & \text { in } & \Gamma_{T} \\
\left.T\right|_{t=0}=T_{\text {ini }} & \text { in } & \Omega_{T}
\end{array}
$$

where,

$$
\left[k_{\text {lam }}\right]=\left[\begin{array}{ccc}
k_{11} & k_{12} & 0 \\
k_{21} & k_{22} & 0 \\
0 & 0 & k_{33}
\end{array}\right]
$$

is the thermal conductivity matrix. 


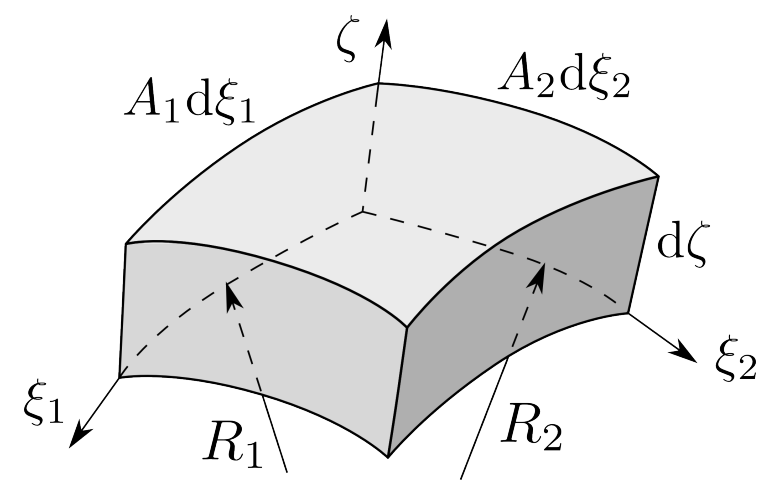

Figure 6. A differential volume in the $\xi_{1} \xi_{2} \zeta$ coordinate system.

Using Galerkin's method [39] multiply the governing equation by an shape function $N$ and integrate by parts,

$$
\begin{aligned}
\int_{V} N\left[(\rho c) \dot{T}-\nabla \cdot\left(\left[k_{\text {lam }}\right] \cdot \nabla T\right)\right] \mathrm{d} V & =0 \\
\int_{V} N(\rho c) \dot{T} \mathrm{~d} V+\int_{V} \nabla N \cdot\left(\left[k_{\text {lam }}\right] \cdot \nabla T\right) \mathrm{d} V & =\int_{\Gamma_{q}} N\left(-\dot{q}_{\text {surf }}\right) \mathrm{d} \Gamma_{q}
\end{aligned}
$$

The choice of the shape function is discussed later.

In the coordinate system $\xi_{1} \xi_{2} \zeta$, as shown in Fig. 6, the gradient operator and the differential volume are, respectively,

$$
\begin{aligned}
\nabla & =\left[\frac{1}{A_{1}} \frac{\partial}{\partial \xi_{1}}, \frac{1}{A_{2}} \frac{\partial}{\partial \xi_{2}}, \frac{\partial}{\partial \zeta}\right] \\
\mathrm{d} V & =A_{1} A_{2} \mathrm{~d} \xi_{1} \mathrm{~d} \xi_{2} \mathrm{~d} \zeta
\end{aligned}
$$

where,

$$
A_{1}=1+\frac{\zeta}{R_{1}}, \quad A_{2}=1+\frac{\zeta}{R_{2}}
$$

Since the shell is thin and shallow,

$$
A_{1} \approx 1, \quad A_{2} \approx 1
$$

Furthermore, assume that the derivatives of temperature w.r.t. $\xi_{1}, \xi_{2}, \zeta$ are the same as derivatives w.r.t. $x, y, z$. Then Eq. (72) and (73) are simplified as,

$$
\begin{aligned}
\nabla & \approx\left[\frac{\partial}{\partial x}, \frac{\partial}{\partial y}, \frac{\partial}{\partial z}\right] \\
\mathrm{d} V & \approx \mathrm{d} x \mathrm{~d} y \mathrm{~d} z
\end{aligned}
$$

Therefore, the terms in Eq. (71) are simplified as,

$$
\begin{gathered}
\int_{V} N(\rho c) \dot{T} \mathrm{~d} V \approx \int_{V} N(\rho c) \dot{T} \mathrm{~d} x \mathrm{~d} y \mathrm{~d} z \\
\int_{V} \nabla N \cdot([k] \cdot \nabla T) \mathrm{d} V \approx \int_{V}\left[\begin{array}{lll}
\frac{\partial N}{\partial x} & \frac{\partial N}{\partial y} & \frac{\partial N}{\partial z}
\end{array}\right]\left[k_{\text {lam }}\right]\left[\begin{array}{c}
\frac{\partial T}{\partial x} \\
\frac{\partial T}{\partial y} \\
\frac{\partial N}{\partial z}
\end{array}\right] \mathrm{d} x \mathrm{~d} y \mathrm{~d} z
\end{gathered}
$$

The analysis outlined above shows:

1. The shell curvatures have very little effect on the thermal solution, i.e. a thin shallow shell can be approximated by a thin flat plate. 
2. The constitutive relations in the $x y z$ coordinate system can be approximated by those in the $\xi_{1} \xi_{2} \zeta$ coordinate system.

Conclusion 1 can be extended to a more general case. Since the shell remains shallow under moderate deflection, shell deformation has very little effect on thermal solution. Therefore, thermal solution of a moderately-deformed thin shallow shell can be approximated by that of an undeformed thin flat plate.

\section{Element matrices and loading vectors}

For each element, the temperature field is interpolated by shape functions $N_{i}, i=1, \cdots, 8$ and nodal values $\mathbf{T}=$ $\left[T_{1}, T_{2}, \cdots, T_{8}\right]^{T}$

$$
T(x, y, z)=L_{4} \mathbf{T}=\left[N_{1}, N_{2}, \cdots, N_{8}\right] \mathbf{T}
$$

The shape functions are,

$$
N_{i+2 j+4 k-6}=f_{i}\left(\eta_{1}\right) f_{j}\left(\eta_{2}\right) f_{k}\left(\eta_{3}\right), \quad i, j, k=1,2
$$

where, $f_{1}(\eta)=\frac{1}{2}(1-\eta), f_{2}(\eta)=\frac{1}{2}(1+\eta)$.

Then the temperature gradient is,

$$
\left[\frac{\partial T}{\partial x}, \frac{\partial T}{\partial y}, \frac{\partial T}{\partial z}\right]^{T}=L_{5} \mathbf{T}
$$

where,

$$
L_{5}=\left[\begin{array}{llll}
N_{1, x} & N_{2, x} & \cdots & N_{8, x} \\
N_{1, y} & N_{2, y} & \cdots & N_{8, y} \\
N_{1, z} & N_{2, z} & \cdots & N_{8, z}
\end{array}\right]
$$

Using approximation provided by Eq. (78) and (79), the first and the second terms in Eq. (71) are discretized as,

$$
\begin{aligned}
\int_{V} N(\rho c) \dot{T} \mathrm{~d} V & \approx\left\{\int_{V} N(\rho c) L_{4} \mathrm{~d} x \mathrm{~d} y \mathrm{~d} z\right\} \dot{\mathbf{T}} \\
\int_{V} \nabla N \cdot([k] \cdot \nabla T) \mathrm{d} V & \approx\left\{\int_{V}\left[\begin{array}{lll}
\frac{\partial N}{\partial x} & \frac{\partial N}{\partial y} & \frac{\partial N}{\partial z}
\end{array}\right]\left[k_{\text {lam }}\right] L_{5} \mathrm{~d} x \mathrm{~d} y \mathrm{~d} z\right\} \mathbf{T}
\end{aligned}
$$

Replacing $N$ using $N_{i}, i=1, \cdots, 8$, the element heat capacity matrix $\left[K_{T}\right]$, thermal conductivity matrix $\left[C_{T}\right]$, and the loading vector $\{Q\}$ are obtained,

$$
\begin{aligned}
{\left[K_{T}\right] } & =\int_{V} L_{5}^{T}\left[k_{\text {lam }}\right] L_{5} \mathrm{~d} V \\
{\left[C_{T}\right] } & =\int_{V} L_{4}^{T}(\rho c) L_{4} \mathrm{~d} V \\
\{Q\} & =\int_{\Omega_{q}} L_{4}^{T}(-\dot{q}) \mathrm{d} \Omega_{q}
\end{aligned}
$$

where,

$$
\dot{q}= \begin{cases}Q, & \text { Constant heat flux } \\ h_{c}\left(T-T_{A W}\right), & \text { Convection } \\ \epsilon \sigma\left(T^{4}-T_{a m b}^{4}\right), & \text { Radiation }\end{cases}
$$

The integrations in Eq. (86)-(88) are evaluated using $2 \times 2 \times 2$ Gaussian quadratures.

\section{Solution to the nonlinear heat transfer problem}

The thermal conductivity and heat capacity matrices and loading vectors of the individual elements are assembled into total thermal conductivity matrix $\left[\bar{K}_{T}\right]$, total heat capacity matrix $\left[\bar{C}_{T}\right]$, total loading vector $\{\bar{Q}\}$, and total temperature vector $\{T\}$. Note that both $\left[\bar{K}_{T}\right]$ and $\left[\bar{C}_{T}\right]$ are nonlinear when the material properties are temperature dependent.

The governing equations for heat transfer problem are,

$$
\left[\bar{C}_{T}(\{T\})\right]\{\dot{T}\}+[\bar{K}(\{T\})]\{T\}=\{\bar{Q}(\{T\})\}
$$

Equation 90 is discretized in the time domain using the Crank-Nicolson scheme [40]. Then Newton-Raphson method is used to solve the resulting nonlinear equations. 
Let $T^{0}=\{T\}_{n}, T^{1}=\{T\}_{n+1}$, and $T^{\alpha}=(1-\bar{\alpha}) T^{0}+\bar{\alpha} T^{1}$,

$$
\left[\bar{C}_{T}\left(T^{\alpha}\right)\right] \frac{T^{1}-T^{0}}{\Delta t}+\left[\bar{K}\left(T^{\alpha}\right)\right] T^{\alpha}=\left\{\bar{Q}\left(T^{\alpha}\right)\right\}
$$

Assuming $T^{0}$ is known, rewrite Eq. (91) as,

$$
\left\{\frac{1}{\Delta t}\left[\bar{C}_{T}\left(T^{\alpha}\right)\right]+\bar{\alpha}\left[\bar{K}\left(T^{\alpha}\right)\right]\right\} T^{1}=\left\{\bar{Q}\left(T^{\alpha}\right)\right\}+\left\{\frac{1}{\Delta t}\left[\bar{C}_{T}\left(T^{\alpha}\right)\right]-(1-\bar{\alpha})\left[\bar{K}\left(T^{\alpha}\right)\right]\right\} T^{0}
$$

To enforce the BC on $\Gamma_{T}$, i.e. temperature is prescirbed on a set of nodes $\tilde{\Gamma}_{T}$,

$$
T_{i}=T_{b c}, \quad i \in \tilde{\Gamma}_{T}
$$

Equation 92 is modified using the penalty method [41],

$$
\left\{\frac{1}{\Delta t}\left[\bar{C}_{T}\left(T^{\alpha}\right)\right]+\bar{\alpha}\left[\bar{K}\left(T^{\alpha}\right)\right]+\left[\bar{K}_{b}\right]\right\} T^{1}=\left\{\bar{Q}\left(T^{\alpha}\right)\right\}+\left\{\frac{1}{\Delta t}\left[\bar{C}_{T}\left(T^{\alpha}\right)\right]-(1-\bar{\alpha})\left[\bar{K}\left(T^{\alpha}\right)\right]\right\} T^{0}+\left\{\bar{Q}_{b}\right\}
$$

where,

$$
\begin{aligned}
{\left[\bar{K}_{b}\right]_{i j} } & = \begin{cases}\tilde{\alpha}, & i=j \in \tilde{\Gamma}_{T} \\
0, & \text { else }\end{cases} \\
\left\{\bar{Q}_{b}\right\}_{i} & = \begin{cases}\tilde{\alpha} T_{b c}, & i \in \tilde{\Gamma}_{T} \\
0, & \text { else }\end{cases}
\end{aligned}
$$

For unconditional stability, let $\bar{\alpha}=\frac{1}{2}$ in the calculation [40]. However, this guarantees unconditional stability in linear systems; and in our case, numerical instability may still appear.

After adding the constraint, rewrite the nonlinear equation Eq. (94),

$$
\left[\bar{C}_{T}\left(T^{\alpha}\right)\right] \frac{T^{1}-T^{0}}{\Delta t}+\left[\bar{K}_{T}\left(T^{\alpha}\right)\right] T^{\alpha}+\left[\bar{K}_{b}\right] T^{1}=\left\{\bar{Q}\left(T^{\alpha}\right)\right\}+\left\{\bar{Q}_{b}\right\}
$$

The Newton-Raphson method is used to solve Eq. (95). In residue form, the method is,

$$
\left[\bar{R}\left(T^{1}\right)\right]=\left[\bar{C}_{T}\left(T^{\alpha}\right)\right] \frac{T^{1}-T^{0}}{\Delta t}+\left[\bar{K}_{T}\left(T^{\alpha}\right)\right] T^{\alpha}+\left[\bar{K}_{b}\right] T^{1}-\left\{\bar{Q}\left(T^{\alpha}\right)\right\}-\left\{\bar{Q}_{b}\right\}
$$

Noting that $\frac{\partial T^{\alpha}}{\partial T^{1}}\{T\}=\bar{\alpha}\{T\}$, the Jacobian of the residue is,

$$
\begin{aligned}
\frac{\partial\left[\bar{R}\left(T^{1}\right)\right]}{\partial T^{1}} & =\frac{\bar{\alpha}}{\Delta t} \underbrace{\frac{\partial\left[\bar{C}_{T}\left(T^{\alpha}\right)\right]}{\partial T^{\alpha}} \Delta \bar{T}}_{\text {Term } 1}+\frac{1}{\Delta t}\left[\bar{C}_{T}\left(T^{\alpha}\right)\right] \\
& +\bar{\alpha} \underbrace{\frac{\partial\left[\bar{K}_{T}\left(T^{\alpha}\right)\right]}{\partial T^{\alpha}} T^{\alpha}}_{\text {Term } 2}+\bar{\alpha}\left[\bar{K}_{T}\left(T^{\alpha}\right)\right]+\left[\bar{K}_{b}\right]-\bar{\alpha} \underbrace{\frac{\partial\left\{\bar{Q}\left(T^{\alpha}\right)\right\}}{\partial T^{\alpha}}}_{\text {Term 3 }}
\end{aligned}
$$

where $\Delta \bar{T}=\{T\}_{n+1}-\{T\}_{n}$.

To obtain the explicit forms that are suitable for programming, the three terms labeled in Eq. (97) need further elaboration. Note that $\left[\bar{C}_{T}\right],\left[\bar{K}_{T}\right]$, and $\{\bar{Q}\}$ are linear combinations of element heat capacity and thermal conductivity matrices and loading vector, respectively. So one can calculate derivatives of element matrices and loading vector and then assemble them to obtain the three terms in Eq. (97).

In each element,

$$
\begin{array}{ll}
\frac{\partial\left[C_{T}\right]}{\partial T^{\alpha}} \Delta T=\left[\cdots\left|\frac{\partial\left[C_{T}\right]}{\partial T_{i}^{\alpha}} \Delta T\right| \cdots\right], & \frac{\partial\left[C_{T}\right]}{\partial T_{i}^{\alpha}}=\int_{V} L_{1}^{T} \frac{\partial(\rho c)}{\partial T_{i}^{\alpha}} L_{1} \mathrm{~d} V \\
\frac{\partial\left[K_{T}\right]}{\partial T^{\alpha}} T^{\alpha}=\left[\cdots\left|\frac{\partial\left[K_{T}\right]}{\partial T_{i}^{\alpha}} T^{\alpha}\right| \cdots\right], & \frac{\partial\left[K_{T}\right]}{\partial T_{i}^{\alpha}}=\int_{V} L_{2}^{T} \frac{\partial\left[k_{l a m}\right]}{\partial T_{i}^{\alpha}} L_{2} \mathrm{~d} V \\
\frac{\partial Q\left(T^{\alpha}\right)}{\partial T^{\alpha}}=\left[\cdots\left|\frac{\partial Q\left(T^{\alpha}\right)}{\partial T_{i}^{\alpha}}\right| \cdots\right], & \frac{\partial Q\left(T^{\alpha}\right)}{\partial T_{i}^{\alpha}}=\int_{\Omega} L_{1}^{T}\left(-\frac{\partial \dot{q}}{\partial T_{i}^{\alpha}}\right) L_{1} \mathrm{~d} \Omega
\end{array}
$$

The derivative of $\dot{q}$ depends on the type of heat flux,

$$
\frac{\partial \dot{q}}{\partial T_{i}^{\alpha}}= \begin{cases}0, & \text { Constant heat flux } \\ h_{c}, & \text { Convection } \\ 4 \epsilon \sigma\left(T_{i}^{\alpha}\right)^{3}, & \text { Radiation }\end{cases}
$$




\section{Coupling Methodology}

\section{A. Coupling scheme for FTSI problems}

\section{Design strategy for the loosely-coupled scheme}

In Ref. [4], a theoretical framework was developed to analyse order of time accuracy of a coupling scheme for FluidStructure Interaction (FSI) problems. The framework is based on a three-field formulation. The formulation includes fluid domain, solid domain, and fluid-mesh-motion domain, and a pseudo-elastodynamic method is used to deform the mesh when solid boundaries move. Note that only the Euler CFD solver based on mesh time-averaging method was considered in the original framework. Therefore for the current study it had to be extended for an NS CFD solver by including the contribution of diffusive flux to the time accuracy of the coupled system.

As pointed out in section I, the numerical stability of the aeroelastic solver in the multicycling scheme can be improved by a proper combination of estimators, i.e. predictors and correctors. The choice of predictor and corrector is facilitated by an energy balance analysis. In Ref. [42], the difference between the aerodynamic work and the sum of kinetic energy and strain energy $E_{t o t}$ in an undamped system is examined by conducting numerical studies. The difference should equal the initial total energy $E_{t o t}$ when the artificial energy of the coupling scheme is zero. The method can be conveniently implemented numerically. However, it cannot be used to determine the order of time accuracy of the artificial energy. In Ref. [6], the energy balance analysis relies on the assumption of harmonic aeroelastic response, i.e. both structural and fluid responses are harmonic. The time accuracy of the artificial energy per period, which is defined as energy accuracy in [6], is analytically determined for the various coupling schemes. However, the energy accuracy defined in this manner cannot be easily verified through numerical experiments, because the validity of theoretical energy accuracy is limited to cases where the aeroelastic responses are harmonic. In current study, a modified energy balance analysis is developed to quantify the energy accuracy. In this analysis framework, energy accuracy is defined as the time accuracy of the artificial energy per time step. In that approach, energy accuracy of a coupling scheme can be determined analytically and verified numerically using arbitrary aeroelastic responses.

Next, two typical loosely coupled schemes for aerothermoelastic simulation are described. Then the framework proposed in Ref. [4] is modified to analyze the time accuracy of a loosely coupled scheme. Subsequently, an energy balance analysis is developed to examine numerical stability of coupling schemes with different combinations of estimators. The first scheme is adapted from the multicycling scheme [2], which will be referred to as the MN scheme. The second scheme is similar to the MN scheme, but with a different set of estimators [6], which will be referred to as the FH scheme. To simplify the energy balance analysis, the subcycling technique discussed in Ref. [2] is not used in the $\mathrm{MN}$ and $\mathrm{FH}$ schemes.

\section{Description of two loosely-coupled schemes}

In a typical aerothermoelastic coupling scheme [2], the solvers are initiated at the same physical time $t$. As the calculations commence, a series of operations are performed to advance the coupled system to next time step.

The coupling procedure of MN scheme is, as illustrated in Fig. 7,

1. Heat flux is passed from the aerodynamic solver to the thermal solver, where heat flux is extrapolated,

$$
\dot{q}^{n+1 / 2, E}=\frac{3}{2} \dot{q}^{n}-\frac{1}{2} \dot{q}^{n-1}
$$

2. Thermal solution is updated to time $t+\Delta t$.

3. Pass new body temperature to structural solver and extrapolate the pressure field by,

$$
p^{n+1, E}=2 p^{n}-p^{n-1}
$$

4. Solve for new displacement $d^{n+1}$ and advance the CSD solver to time $t+\Delta t$.

5. Pass new wall temperature and new displacement to aerodynamic solver by,

$$
d^{n+1, E}=d^{n+1}
$$

6. Advance aerodynamic solver to time $t+\Delta t$.

7. Above steps are repeated until end of the simulation.

The coupling procedure of FH scheme is, as illustrated in Fig. 8,

1. Heat flux is passed from the aerodynamic solver to the thermal solver, where heat flux is extrapolated,

$$
\dot{q}^{n+1 / 2, E}=\frac{3}{2} \dot{q}^{n}-\frac{1}{2} \dot{q}^{n-1}
$$


2. Thermal solution is updated to time $t+\Delta t$.

3. Pass new wall temperature to aerodynamic solver and extrapolate displacements by,

$$
d^{n+1, E}=d^{n}+\Delta t\left(\frac{3}{2} \dot{d}^{n}-\frac{1}{2} \dot{d}^{n-1}\right)
$$

4. Solve for new pressure field $p^{n+1}$ and advance the aerodynamic solver to time $t+\Delta t$.

5. Pass new body temperature and new pressure field to structural solver by,

$$
p^{n+1, E}=p^{n+1}
$$

6. Advance the structural solver to $t+\Delta t$.

7. Above steps are repeated until end of the simulation.

For both aeroelastic schemes, a starting procedure is needed. In current study, following procedure is employed [43],

$$
\begin{aligned}
& \dot{d}^{1}=\dot{d}^{0}+\Delta t \ddot{d}^{0} \\
& d^{1}=d^{0}+\Delta t \dot{d}^{0}+\frac{1}{2} \Delta t^{2} \ddot{d}^{0}
\end{aligned}
$$

Subsequently the loading at step 1 is calculated using $d^{1}$ and $\dot{d}^{1}$. The same starting procedure is used for both schemes so that their time accuracy can be compared on the basis.

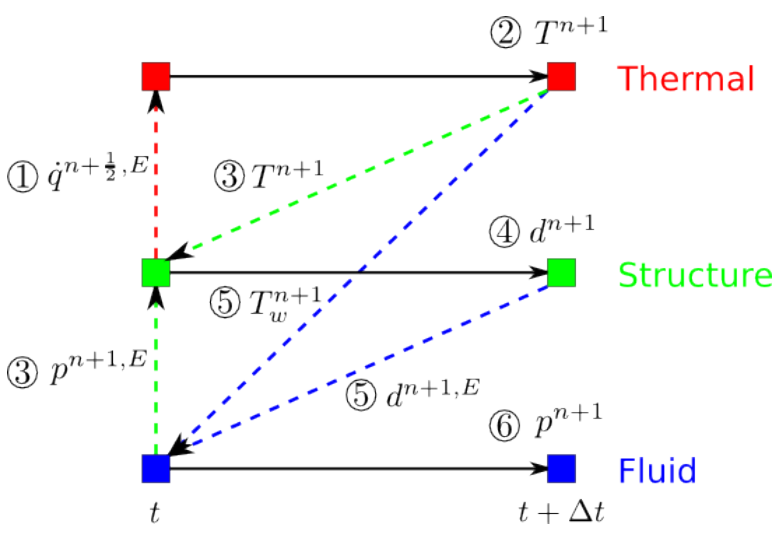

Figure 7. The coupling procedure for MN scheme.

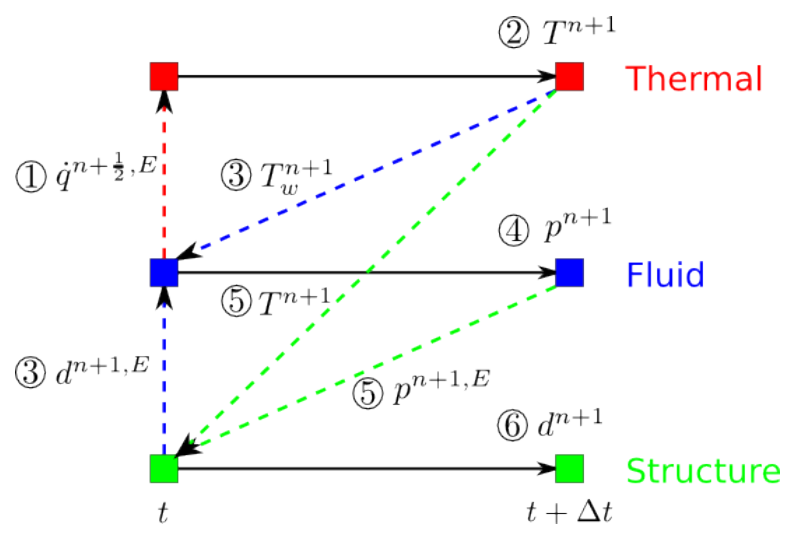

Figure 8. The coupling procedure for FH scheme.

\section{Time accuracy analysis}

In aeroelastic simulations, the structural and aerodynamic solvers constructed using second-orer time accurate schemes may not demonstrate the same order of time accuracy in a loosely-coupled scheme [4]. Therefore, it is a question whether the thermal, structural, and aerodynamic solvers in current study remains second-order time accurate in the loosely-coupled scheme. In the following, the analysis approach from Ref. [4] is adapted to determine the conditions under which the loosely-coupled scheme is second-order time accurate for aerothermoelastic simulations.

AERodynamic SOlVER The discretized form of Eq. (1) when dealing with the isolated fluid domain is given in Eq. (5). However, in the coupled system, the boundary displacements, and thus the sequences of meshes, are approximated by a displacement estimator. Furthermore, the wall temperature is provided by a thermal solver. Therefore, the discretized CFD solver in the coupled scheme is,

$$
\begin{aligned}
\frac{1}{\Delta t}\left[\frac{3}{2}\left(V^{E} u_{f}\right)_{i}^{n+1}-2\left(V^{E} u_{f}\right)_{i}^{n}\right. & \left.+\frac{1}{2}\left(V^{E} u_{f}\right)_{i}^{n-1}\right]+F\left(u_{f}^{n+1}, \chi^{n+1, E}, \chi^{n, E}, \chi^{n-1, E}, T_{w}^{n+1}\right) \\
& =G\left(u_{f}^{n+1}, \chi^{n+1, E}, \chi^{n, E}, \chi^{n-1, E}, T_{w}^{n+1}\right)
\end{aligned}
$$


Next, consider the error in Eq. (108) compared with Eq. (5),

$$
\epsilon_{C F D}=\epsilon_{u}+\epsilon_{F}+\epsilon_{G}
$$

where, $\epsilon_{u}, \epsilon_{F}, \epsilon_{G}$ are numerical errors from time derivative, convective flux, and diffusive flux terms, respectively,

$$
\begin{aligned}
\epsilon_{u} & =\frac{1}{\Delta t}\left[\frac{3}{2}\left(V^{E} u_{f}\right)^{n+1}-2\left(V^{E} u_{f}\right)^{n}+\frac{1}{2}\left(V^{E} u_{f}\right)^{n-1}\right]-\frac{1}{\Delta t}\left[\frac{3}{2}\left(V^{E} u_{f}\right)^{n+1}-2\left(V^{E} u_{f}\right)^{n}+\frac{1}{2}\left(V^{E} u_{f}\right)^{n-1}\right] \\
& =O\left(\left|\chi^{n+1, E}-\chi^{n+1}\right|\right) \\
\epsilon_{F} & =F\left(u_{f}^{n+1}, \chi^{n+1, E}, \chi^{n, E}, \chi^{n-1, E}, T_{w}^{n+1}\right)-F\left(u_{f}^{n+1}, \chi^{n+1}, \chi^{n}, \chi^{n-1}, T_{w}^{n+1}\right) \\
& =\sum_{k=-1}^{1} O\left(\left|\chi^{n+k, E}-\chi^{n+k}\right|\right) \\
\epsilon_{G} & =G\left(u_{f}^{n+1}, \chi^{n+1, E}, \chi^{n, E}, \chi^{n-1, E}, T_{w}^{n+1}\right)-G\left(u_{f}^{n+1}, \chi^{n+1}, \chi^{n}, \chi^{n-1}, T_{w}^{n+1}\right) \\
& =\sum_{k=-1}^{1} O\left(\left|\chi^{n+k, E}-\chi^{n+k}\right|\right)
\end{aligned}
$$

Therefore the error due to approximation of boundary displacements is,

$$
\begin{aligned}
\epsilon_{C F D} & =\sum_{k=-1}^{1} O\left(\left|\chi^{n+k, E}-\chi^{n+k}\right|\right) \\
& =\sum_{k=-1}^{1} O\left(\left|d^{n+k, E}-d^{n+k}\right|\right)
\end{aligned}
$$

Since Eq. (108) is second-order time-accurate, when $O\left(\left|\chi^{n+k, E}-\chi^{n+k}\right|\right)=O\left(\Delta t^{2}\right)$, i.e. displacement estimator is at least second-order time-accurate, and therefore the time accuracy of CFD solver in coupled scheme is preserved. Note that as long as the updated wall temperature is passed to aerodynamic solver, thermal solution will not effect the time-accuracy of the aerodynamic solution.

STRUCTURAL SOLVER From Eq. (52), the governing equation for structural domain is,

$$
[M] \ddot{d}^{n+1}+[C] \dot{d}^{n+1}+\left[K\left(d^{n+1}, T^{n+1}\right)\right] d^{n+1}=\left\{F\left(p^{n+1}, d^{n+1}, \dot{d}^{n+1}, T^{n+1}\right)\right\}
$$

Using the Newmark- $\beta$ scheme for time integration yields,

$$
\begin{aligned}
& d^{n+1}=d^{n}+\dot{d}^{n} \Delta t+\frac{\Delta t^{2}}{4}\left(\ddot{d}^{n+1}+\ddot{d}^{n}\right) \\
& \dot{d}^{n+1}=\dot{d}^{n}+\frac{\Delta t}{2}\left(\ddot{d}^{n+1}+\ddot{d}^{n}\right)
\end{aligned}
$$

In coupled system, the loading $p^{n+1}$ is provided by an estimator, such that

$$
\left\{F\left(p^{n+1}, d^{n+1}, \dot{d}^{n+1}, T^{n+1}\right)\right\} \approx\left\{F\left(p^{n+1, E}, d^{n+1}, \dot{d}^{n+1}, T^{n+1}\right)\right\}
$$

Then the discretization error is,

$$
\epsilon_{C S D}=\epsilon_{d}+\epsilon_{f}
$$

where $\epsilon_{d}$ is the error associated with Newmark- $\beta$ scheme and $\epsilon_{f}$ is the error associated with loading estimation,

$$
\begin{aligned}
\epsilon_{d} & =\left\{[M] \ddot{d}^{n+1}+[C] \dot{d}^{n+1}+\left[K\left(d^{n+1}, T^{n+1}\right)\right] d^{n+1}\right\} \\
& -\left\{[M] \ddot{d}^{n+1}+[C]\left[\dot{d}^{n}+\frac{\Delta t}{2}\left(\ddot{d}^{n+1}+\ddot{d}^{n}\right)\right]+[K]\left[d^{n}+\dot{d}^{n} \Delta t+\frac{\Delta t^{2}}{4}\left(\ddot{d}^{n+1}+\ddot{d}^{n}\right)\right]\right\} \\
& =O\left(\Delta t^{2}\right) \\
\epsilon_{f} & =\left\{F\left(p^{n+1, E}, d^{n+1}, \dot{d}^{n+1}, T^{n+1}\right)\right\}-\left\{F\left(p^{n+1}, d^{n+1}, \dot{d}^{n+1}, T^{n+1}\right)\right\} \\
& =O\left(\left|p^{n+1, E}-p^{n+1}\right|\right)
\end{aligned}
$$


The total error due to discretization in the coupled scheme is,

$$
\epsilon_{C S D}=O\left(\left|p^{n+1, E}-p^{n+1}\right|\right)+O\left(\Delta t^{2}\right)
$$

Therefore when the pressure estimator is at least second-order time-accurate, the CSD solver in coupled scheme remains second-order time-accurate. Similarly to the the aerodynamic solver, the temperature does not effect the time-accuracy of the solver.

THERMAL SOLVER From Eq. (90), the governing equation for thermal domain is,

$$
[C] \dot{T}^{n+1 / 2}+\left[K\left(T^{n+1 / 2}\right)\right] T^{n+1 / 2}=\dot{q}^{n+1 / 2}
$$

Using the Crank-Nicolson scheme for time integration yields,

$$
\dot{T}^{n+1 / 2}=\frac{T^{n+1}-T^{n}}{\Delta t}
$$

In coupled system, the heat flux $\dot{q}^{n+1 / 2}$ is provided by an estimator from the CFD solver. Then the discretization error is,

$$
\epsilon_{C T D}=\epsilon_{T}+\epsilon_{q}
$$

where $\epsilon_{T}$ is the error associated with Crank-Nicolson scheme and $\epsilon_{q}$ is the error associated with heat flux estimation,

$$
\begin{aligned}
\epsilon_{T} & =\left\{[C] \dot{T}^{n+1 / 2}+\left[K\left(T^{n+1 / 2}\right)\right] T^{n+1 / 2}\right\}-\left\{[C] \frac{T^{n+1}-T^{n}}{\Delta t}+\left[K\left(T^{n+1 / 2}\right)\right] T^{n+1 / 2}\right\} \\
& =O\left(\Delta t^{2}\right) \\
\epsilon_{q} & =O\left(\left|\dot{q}^{n+1 / 2, E}-\dot{q}^{n+1 / 2}\right|\right)
\end{aligned}
$$

The total error due to discretization in the coupled scheme is,

$$
\epsilon_{C T D}=O\left(\left|\dot{q}^{n+1 / 2, E}-\dot{q}^{n+1 / 2}\right|\right)+O\left(\Delta t^{2}\right)
$$

Therefore as long as the heat flux estimator is at least second-order time-accurate, the CTD solver in coupled scheme remains second-order time-accurate.

SUMMARY OF TIME ACCURACY ANALYSIS Based on above discussion, the loosely coupled scheme for aerothermoelastic simulation is second-order time-accurate when,

1. The aerodynamic, structural, and thermal solvers are second-order time-accurate.

2. The displacement, force, and heat flux estimators are second-order time-accurate.

It is obvious that the aerothermoelastic coupling procedures using $\mathrm{MN}$ and $\mathrm{FH}$ schemes both produce second-order time-accurate solutions.

\section{The energy balance analysis}

The aeroelastic schemes can be further characterized using energy balance analysis. In Ref. [6], the work done on the structure by fluid $\delta E^{S}$ in a second-order time-accurate structural solver is,

$$
\delta E^{S}=\frac{1}{2}\left(p^{n+1, E}+p^{n, E}\right)\left(d^{n+1}-d^{n}\right)
$$

and the work done on the fluid by structure $\delta E^{S}$ in a second-order time-accurate fluid solver is,

$$
\delta E^{F}=-\frac{1}{2}\left(p^{n+1}+p^{n}\right)\left(d^{n+1, E}-d^{n, E}\right)
$$

The artificial energy $\delta E$ over one time step is,

$$
\delta E=\delta E^{S}+\delta E^{F}
$$


It is evident that the artificial energy is non-zero unless

$$
\begin{aligned}
& p^{n+1, E}=p^{n+1} \\
& d^{n+1, E}=d^{n+1}
\end{aligned}
$$

However, in loosely-coupled schemes, the values of $d^{n+1}$ and $p^{n+1}$ are not known at the same time and either $d^{n+1}$ or $p^{n+1}$ is approximated. Therefore, in loosely-coupled schemes, artificial energy accumulates at each time step, resulting in error accumulation and eventual numerical instability.

The artificial energy can be quantified by its order of time accuracy. Using the following combination of estimators as example,

$$
\begin{aligned}
& p^{n+1, E}=2 p^{n}-p^{n-1} \\
& d^{n+1, E}=d^{n+1}
\end{aligned}
$$

which corresponds to the case in the MN scheme [2]. Expanding $\delta E$ in Taylor series at the $n$th time step,

$$
\begin{aligned}
\delta E & =\frac{1}{2}\left(p^{n}+\frac{1}{2} p^{n-1}-\frac{1}{2} p^{n-2}\right)\left(d^{n+1}-d^{n}\right)-\frac{1}{2}\left(p^{n+1}+p^{n}\right)\left(d^{n+1}-d^{n}\right) \\
& =-\ddot{p}^{n} \dot{d}^{n} \Delta t^{3}=O\left(\Delta t^{3}\right)
\end{aligned}
$$

Therefore, the artificial energy in the MN scheme is third-order time-accurate. For simplicity, we refer to a scheme as $p$ th-order energy-accurate when its artificial energy per time step is $p$ th-order time-accurate.

On the other hand, if the estimators indicated in [6] are used,

$$
\begin{aligned}
& p^{n+1, E}=p^{n+1} \\
& d^{n+1, E}=d^{n}+\Delta t\left(\frac{3}{2} \dot{d}^{n}-\frac{1}{2} \dot{d}^{n-1}\right)
\end{aligned}
$$

The scheme is clearly second-order time-accurate. Furthermore, energy balance analysis shows, the scheme is fourthorder energy-accurate with

$$
\delta E=\frac{5}{12} p^{n} u^{(4), n} \Delta t^{4}=O\left(\Delta t^{4}\right)
$$

which is one-order higher than that of the MN scheme.

It is worthwhile pointing out the relation between energy-accuracy and damping of the system. In an FSI system, assume the structural displacements are,

$$
d=\bar{d} e^{(-\zeta+i \omega) t}=\bar{d} e^{z t}
$$

The sum of kinetic energy and strain energy of the structure is,

$$
\begin{aligned}
E_{t o t} & =\frac{1}{2} d^{T}[K] d+\frac{1}{2} \dot{d}^{T}[M] \dot{d} \\
& =\frac{1}{2}\left(\bar{d}^{T}[K] \bar{d}+\bar{d}^{T}[M] \bar{d} z^{2}\right) e^{2 z t} \\
& =\bar{E}_{t o t} e^{2 z t+\phi(z)}
\end{aligned}
$$

The expressions of total energy $E_{t o t}$ in two consecutive time steps are,

$$
\begin{aligned}
E_{t o t}^{n} & =\bar{E}_{t o t} e^{2 z t+\phi(z)} \\
E_{t o t}^{n+1} & =\bar{E}_{t o t} e^{2 z(t+\Delta t)+\phi(z)}
\end{aligned}
$$

The energy gained or lost during one time step is,

$$
\begin{aligned}
\Delta E_{t o t} & =E_{t o t}^{n+1}-E_{t o t}^{n}=\bar{E}_{t o t} e^{2 z t+\phi(z)}\left(e^{2 z \Delta t}-1\right) \\
& =\bar{E}_{t o t} e^{2 z t+\phi(z)}\left[2 z \Delta t+O\left(\Delta t^{2}\right)\right]
\end{aligned}
$$

On the other hand, in a loosely-coupled scheme with $p$ th-order energy-accuracy, $\Delta E_{t o t}=O\left(\Delta t^{p}\right)$. Therefore,

$$
z e^{2 z t+\phi(z)}=O\left(\Delta t^{p-1}\right)
$$


Consider the real part of Eq. (144),

$$
\zeta e^{-2 \zeta t+\phi(\zeta)}=O\left(\Delta t^{p-1}\right)
$$

If the FSI system is close to flutter point, $E_{\text {tot }}$ tends to be constant and thus $\zeta \rightarrow 0$. Then,

$$
\begin{aligned}
\zeta e^{-2 \zeta t+\phi(\zeta)} & =\zeta+O\left(\zeta^{2}\right) \\
\zeta & =O\left(\Delta t^{p-1}\right)
\end{aligned}
$$

Therefore we can conclude that: When a loosely-coupled partitioned scheme is pth-order energy-accurate, the damping coefficient is (p-1)th-order time-accurate near flutter point. Therefore loosely-coupled schemes with higher-order energy-accuracy predict the damping coefficient, and thus the flutter point, with higher time accuracy.

\section{Verification of the Framework}

\section{A. Verification of aerodynamic solver}

1. The test case considered

Due to the lack of experimental data for unsteady aerodynamics in hypersonic flow, a case of pitching NACA0012 airfoil oscillating in pitching is used to verify the correctness and time accuracy of the aerodynamic solver. For the cases of pitching airfoil used in aeroelastic research, there are several data sets available from experimental studies and numerical simulations in the literature. One of the Computational Test (CT) cases, namely CT1, published in AGARD report 702 [44] is selected to validate the RANS solution obtained from the CFD solver. The test conditions are provided in Table 1 . The NACA0012 airfoil oscillates in pitching about quarter-chord and the motion is given by,

$$
\alpha=\alpha_{m}+\alpha_{a} \sin (\omega t)
$$

Table 1. Setup of the test cases

\begin{tabular}{ccccc}
\hline$\alpha_{m}\left({ }^{\circ}\right)$ & $\alpha_{a}\left(^{\circ}\right)$ & $R e\left(\times 10^{6}\right)$ & $M$ & $k$ \\
\hline 2.77 & 2.34 & 4.8 & 0.6 & 0.0808 \\
\hline
\end{tabular}

\section{Unsteady results}

For unsteady simulations, the physical time step is $0.01 \mathrm{~s}$. For each time step, up to 2000 subiterations in the CFD solver are used to march the fluid in time. Six cycles of oscillation are completed to remove the effect of initial conditions. The results from CT1 case are shown in Figs. 9-10, where CFD data from McMullen [45] and Pandya [46] are included for comparison. The results from SUmb agree well with experimental data and other CFD results for the time history of the lift coefficient as shown in Fig. 9. When considering the moment coefficient shown in Fig. 10, all the CFD data agree well with each other, but are not sufficiently close to the experimental data. In the experiment, the surface shear stress is missing, which could be the source of the disagreement between experimental and computational data. Therefore, it was concluded that the ALE formulation in SUmb is implemented correctly. 


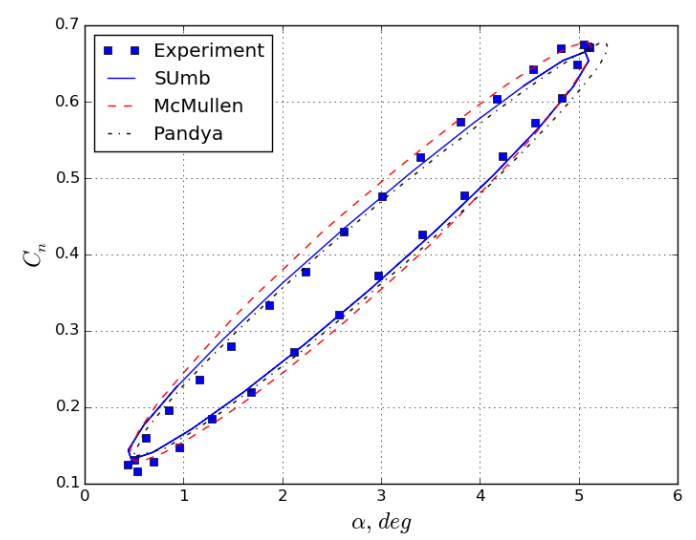

Figure 9. Lift coefficient as a function of instantaneous angle of attack in $\mathrm{CT} 1$ case.

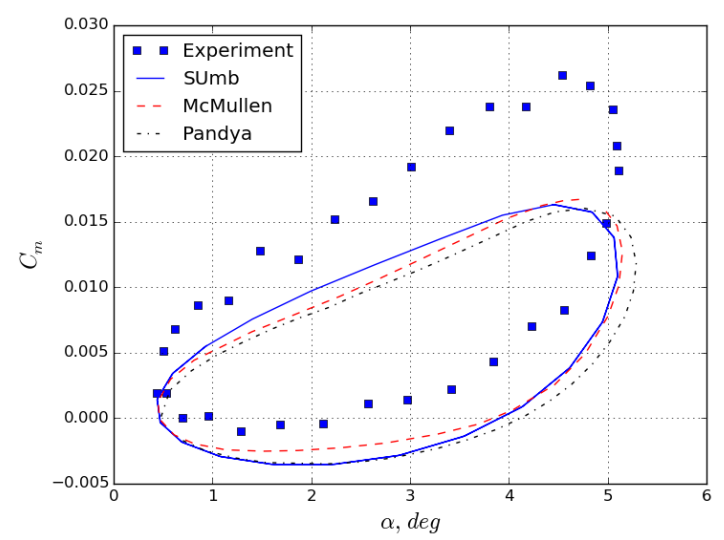

Figure 10. Moment coefficient as a function of instantaneous angle of attack in CT1 case.

\section{Time accuracy}

Assuming that the CFD solver has $p$ th order temporal accuracy and $q$ th order spatial accuracy, and a solution $u_{\Delta t, \chi}$ is obtained at time step $\Delta t$ and a given mesh $\chi$, the error compared to the exact solution $\tilde{u}$ is,

$$
\epsilon_{\Delta t, \chi}=u_{\Delta t, \chi}-\tilde{u}=\Psi_{1} \Delta t^{q_{1}}+\epsilon_{\chi}
$$

where $\Psi_{1}$ is a constant dependent on the numerical scheme, and $\epsilon_{\chi}$ is error from spatial discretization and assumed to be independent of time step. Using the same mesh and generating solutions $u_{i}$ over a series of increasing time steps $k_{i} \Delta t$, e.g. $\Delta t, 2 \Delta t, 4 \Delta t, \cdots$ and using the solution $u_{0}$ of the smallest time step $t$ as reference, the error in time discretization can be expressed using $l^{2}$ norm as,

$$
\epsilon_{\Delta} t=\left\|u_{i}-u_{0}\right\|_{l^{2}}=\left\|\Psi_{1}\left(1-\left(\frac{1}{k_{i}}\right)^{q}\right)\right\|\left(k_{i} \Delta t\right)^{q}=\Psi\left(k_{i} \Delta t\right)^{q}
$$

where $q$ is order of accuracy. When $k_{i}$ is sufficiently large,

$$
\Psi \approx\left\|\Psi_{1}\right\|
$$

and

$$
\log \epsilon_{\Delta t}=\log \Psi+q \log \left(k_{i} \Delta t\right)
$$

Therefore, in the log plot of relative error vs. time step, the slope of the line indicates the order of time accuracy of the solutions.

The CFD solutions at $t=0.6 \mathrm{~s}$ are obtained at a series of time steps: $\Delta t=0.02 \mathrm{~s}, 0.01 \mathrm{~s}, 0.005 \mathrm{~s}, 0.002 \mathrm{~s}$. Using the solution at $\Delta t=0.002 \mathrm{~s}$ as reference, the error plot is shown in Fig. 11. The result indicates the CT1 solutions are second-order time accurate. The mathematical formulation and code implementation in SUmb is identical for subsonic and hypersonic flow regimes, therefore above results indicate that the aerodynamic solver is second-order time accurate for hypersonic flow regime. 


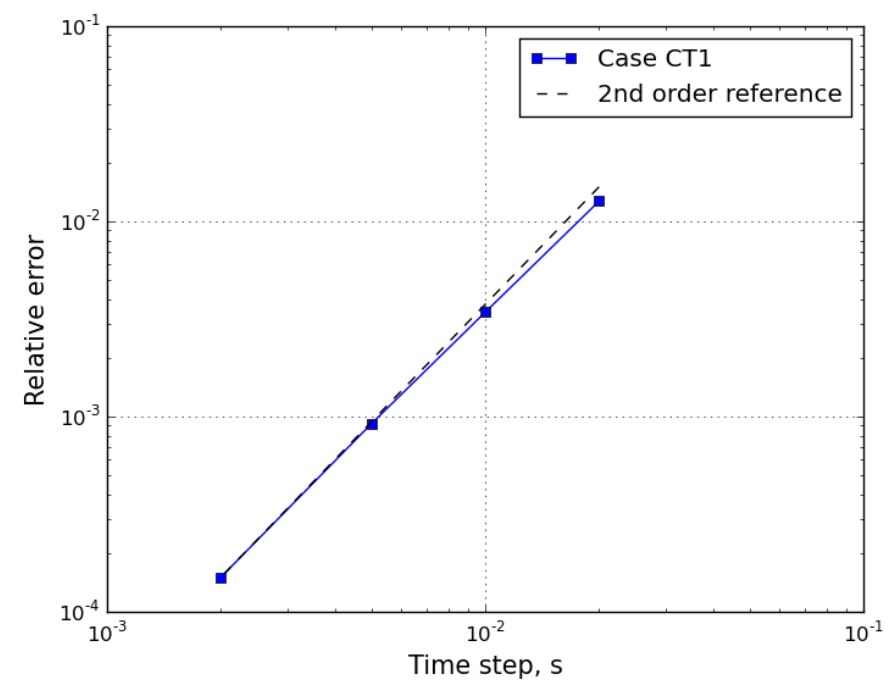

Figure 11. The log plot of relative error v.s. time step for CT1.

\section{B. Verification of structural solver}

The structural solver is verified using several cases for panel flutter. The data for comparison are collected from the literature (Nydick et al. [47], Dowell [48], and Gray et al. [49]). Two sets of material parameters are shown in Table 2. The test case considered are shown in Table 3. In the last case, the temperature increments are $\Delta T=T_{c r}, 2 T_{c r}$. The thermal loading is modelled by Eq. (64), and the aerodynamic loading is modelled by piston theory using Eq. (66).

Table 2. Nondimensionalized material properties

\begin{tabular}{cccccccccc}
\hline Set & $E_{1}$ & $E_{2}$ & $\nu$ & $G_{12}$ & $G_{13}$ & $G_{23}$ & $\rho$ & $\alpha_{1}, 10^{-6}$ & $\alpha_{2}, 10^{-6}$ \\
\hline 1 & 1 & 1 & 0.3 & 0.3846 & 0.3846 & 0.3846 & 1 & 1.2 & 1.2 \\
2 & 1 & 0.6452 & 0.27 & 0.2710 & 0.2710 & 0.2710 & 1 & 3.22 & 10.6 \\
\hline
\end{tabular}

Table 3. Data for the cases of panel flutter

\begin{tabular}{ccccc}
\hline Case & Geometry & Material & BC & Loading \\
\hline Isotropic plate & $a=b=240, h=1$ & 1 & Simply supported & $M_{\infty}=10, \mu=1$ \\
Orthotropic plate & $a=b=300, h=1$ & 2 & Simply supported & $M_{\infty}=15, \mu=1.5$ \\
Heated Isotropic plate & $a=b=500, h=1$ & 1 & Simply supported & $M_{\infty}=10, \mu=5.355 \times 10^{-3}$ \\
\hline
\end{tabular}

In general, the results produced by DCS9 agree well with those from literature. In the first case (Fig. 12), the predicted flutter points are almost identical. In the second case (Fig. 12), DCS9 overpredicts the flutter point by about $1 \%$. In both cases, the limit cycle curves agree quite well.

In the third case (Fig. 13), when $\Delta T=T_{c r}$, DCS9 underpredicts the flutter point by approximately $2.9 \%$. However, when $\Delta T=2 T_{c r}$, results from DCS9 suggest there is no flutter point. Instead, initially the panel is thermally buckled, which is dynamically stable. When $\lambda \geq 200$, the panel enters a chaotic region, where no regular limit cycle can be identified. When $\lambda \geq 250$, the panel finally reaches an limit cycle oscillation. 


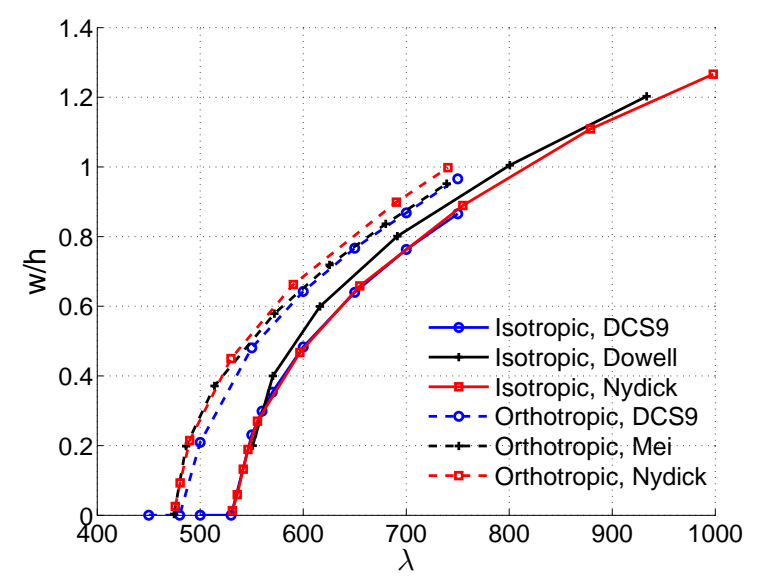

Figure 12. Isotropic and orthotropic plates.

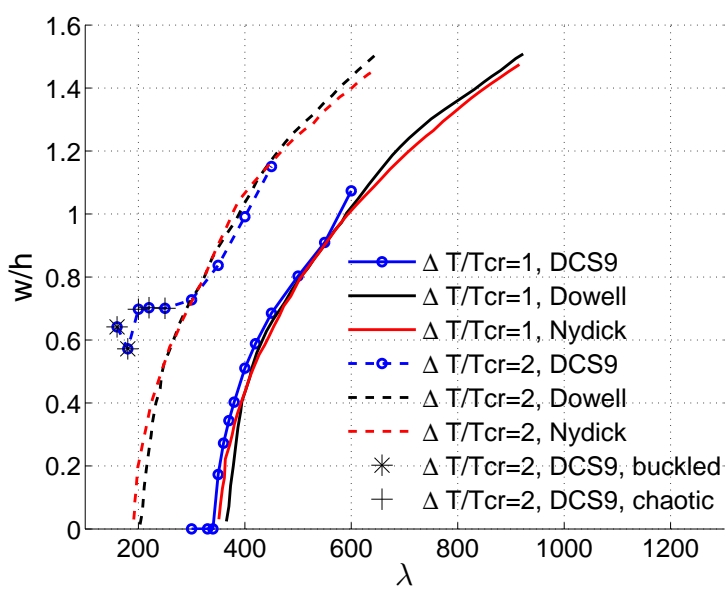

Figure 13. Heated isotropic plate.

\section{Verification of thermal solver}

The thermal solver is verified by examining heat transfer in a generic skin panel exposed to aerodynamic heating.

The layers of SITPS-based generic skin panel is shown in Fig. 14. The skin panel is composed of corrugated sandwich structure with insulated core material. The choice of material is based on Ref. [50]. A type of ceramic matrix composite, AS/N720, is used for the outer panel (i.e. hot structure) and the web. Beryllium is used for inner panel, i.e. cold structure. The core is filled with Saffil as insulator. The material properties at $300 \mathrm{~K}$ are shown in Table 4. The original design of SITPS in Ref. [50] is for rocket-based vehicle, which can have a thickness of 140mm. In the generic skin panel, total thicknesses of layers are decreased to $12 \mathrm{~mm}$ to be applicable to a hypersonic air-breathing vehicle.

A cylindrical shell, shown in Fig. 15, is used to demonstrate the capability of the heat transfer model. The geometric parameters of the shell are provided in Table 5. The composite layup is shown in Table 4. The material properties are temperature dependent, unless otherwise stated.

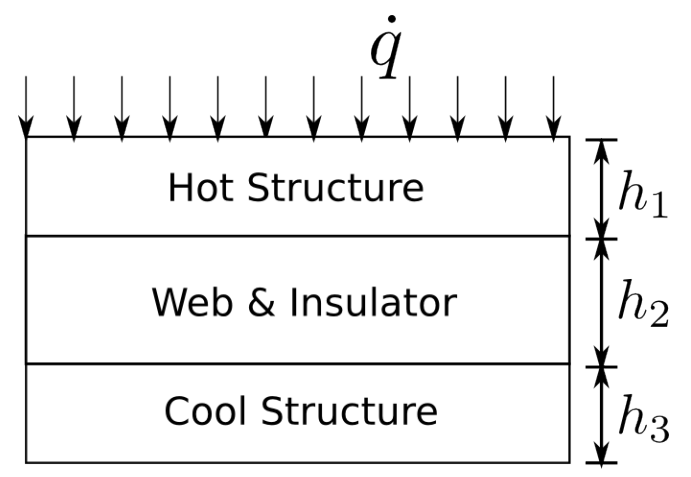

Figure 14. A generic skin panel.

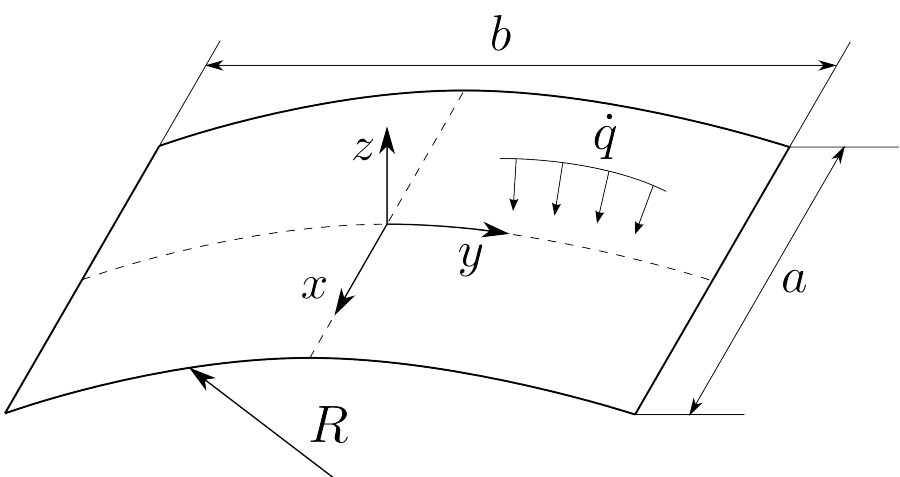

Figure 15. A cylindrical shell.

The shell is insulated on the bottom and the four sides. Heat loading is applied only to the upper surface. Initially, the temperature of shell is uniform at $T=329.82 \mathrm{~K}$. Uniform convective heating and radiation are applied to the upper surface. The heat flux is,

$$
\dot{q}=h_{c}\left(T-T_{A W}\right)+\epsilon \sigma\left(T^{4}-T_{a m b}^{4}\right)
$$

where $h_{c}=6.03 \mathrm{~W} / \mathrm{m}^{2} / \mathrm{K}, T_{A W}=1317.02 \mathrm{~K}, T_{a m b}=329.82 \mathrm{~K}$, and $\epsilon=0.85$. The heat load is applied for 600s. 
Table 4. Material properties for generic skin panel

\begin{tabular}{ccccc}
\hline & $\rho, \mathrm{kg} / \mathrm{m}^{3}$ & $k_{h}, \mathrm{~J} / \mathrm{m} / \mathrm{K}$ & $c, \mathrm{~J} / \mathrm{kg} / \mathrm{K}$ & $h_{i}, \mathrm{~mm}$ \\
\hline AS/N720 [51] & 2600 & 2.47 & 786.8 & 2.0 \\
Saffil [52] & 49.98 & 0.0258 & 942 & 7.0 \\
Beryllium [53] & 1855 & 173.6 & 1895 & 3.0 \\
\hline
\end{tabular}

Table 5. Geometry parameters of the shell

\begin{tabular}{ccc}
\hline$a$ & $b$ & $R$ \\
\hline $508 \mathrm{~mm}$ & $508 \mathrm{~mm}$ & $2540 \mathrm{~mm}$ \\
\hline
\end{tabular}

The HTSH element is compared with the DS3 element from commercial software ABAQUS, which is a three-node triangular shell element [36]. In FE models based on the DS3 element, 7 unknown temperatures are assigned to each layer of the shell. The unknowns at interfaces are shared by neighboring layers and thus each node has a total of 19 unknown temperatures in the thickness direction. Note that in the ABAQUS models, the geometry model of the shell is curved and thus the effect of curvature is included. In the HTSH models, the effect of curvature is neglected.

The solutions at $t=300 \mathrm{~s}$ and $t=600 \mathrm{~s}$ using the HTSH and DS3 elements are shown in Fig. 16 and 17.

In Fig. 16, when material properties are temperature independent, the temperature distribution is almost piecewisely linear. The HTSH and DS3 solutions match very well with each other, with maximum error less than $1 \%$. Therefore, neglecting curvature in the HTSH element does not affect the accuracy of the thermal solution.

In Fig. 17, temperature dependence of material properties speeds up the heating of the inner panel and causes nonlinear temperature distribution in the thickness direction. However, the distribution is still approximated well by linear interpolation using HTSH element, producing a maximum error of $1.2 \%$. Therefore, the HTSH element captures the through-the-thickness temperature distribution in the presence of nonlinear heat loading and temperaturedependent material properties.

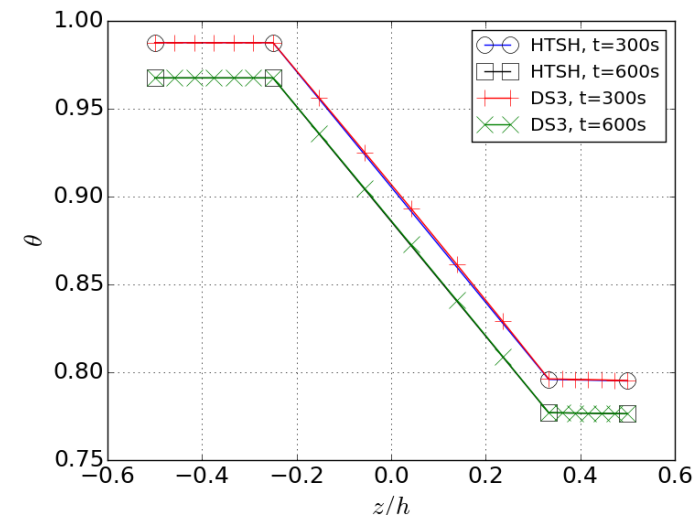

Figure 16. Solution of Case 2 with material set 1.

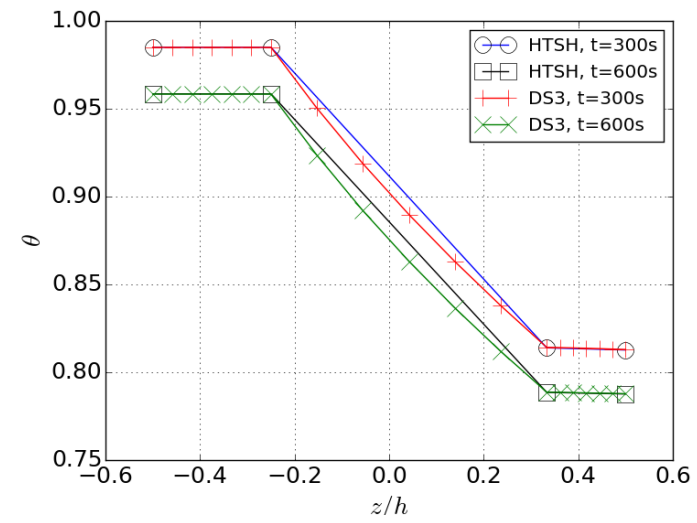

Figure 17. Solution of Case 2 with material set 2.

\section{Verification of aeroelastic coupling scheme}

\section{Test case considered}

The flutter of typical section consisting of a double-wedge airfoil in hypersonic flow, shown in Fig. 18. The purpose of the test case is to examine the time accuracy and energy accuracy of the coupling schemes, which requires intensive computational efforts associated with very fine time steps. Therefore, the Euler CFD solver is employed to speed up the verification process.

The governing equations of the aeroelastic system are,

$$
\left[\begin{array}{cc}
m & S_{\alpha} \\
S_{\alpha} & I_{\alpha}
\end{array}\right]\left[\begin{array}{c}
\ddot{h} \\
\ddot{\alpha}
\end{array}\right]+\left[\begin{array}{cc}
K_{h} & 0 \\
0 & K_{\alpha}
\end{array}\right]\left[\begin{array}{l}
h \\
\alpha
\end{array}\right]=\left[\begin{array}{c}
-L \\
M_{e a}
\end{array}\right]
$$


The case is adapted from [12], and the parameters are given in Table 6.

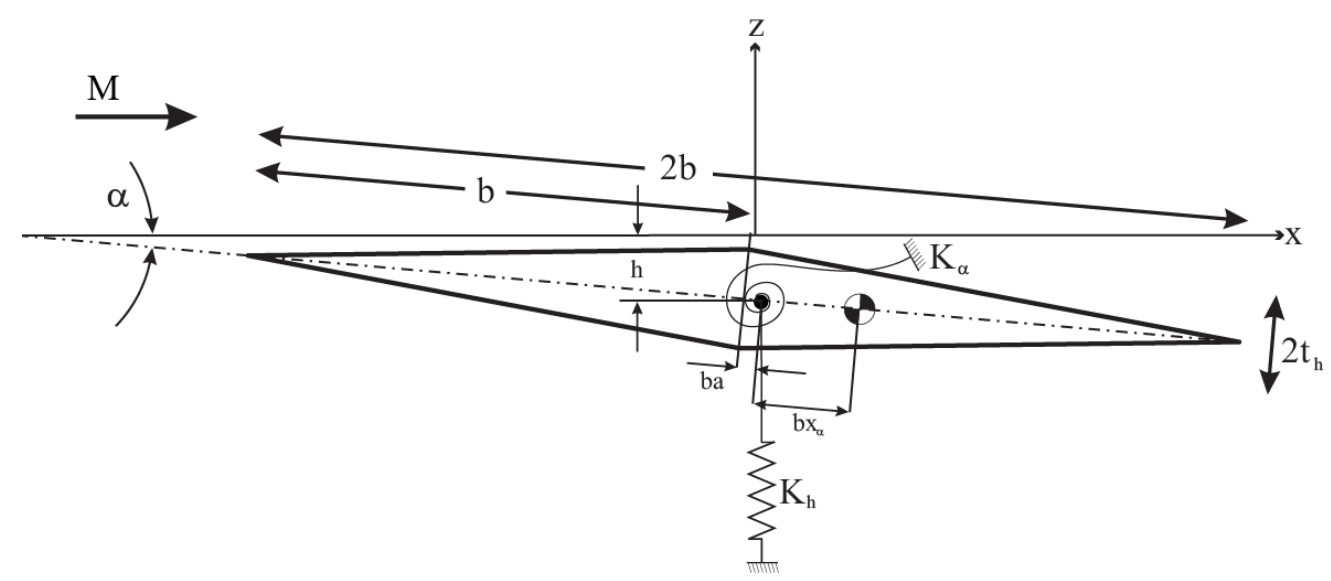

Figure 18. Case setup of the typical section flutter [12].

Table 6. Parameters of the aeroelastic system.

\begin{tabular}{cccccccc}
\hline$m$ & $S_{\alpha}$ & $I_{\alpha}$ & $K_{h}$ & $K_{\alpha}$ & $b$ & $t_{h} / b$ & $\alpha_{S}$ \\
\hline $94.274 \mathrm{~kg}$ & $22.154 \mathrm{kgm}$ & $30.499 \mathrm{kgm}^{2}$ & $668284.31 \mathrm{~N} / \mathrm{m}$ & $1702262.79 \mathrm{Nm} / \mathrm{rad}$ & $1.175 \mathrm{~m}$ & 0.0336 & $0^{\circ}$ \\
\hline
\end{tabular}

The flight conditions are, $H=12000 \mathrm{~m}, M_{\infty}=13, a=0.0$, and in this case, the system is close to flutter point. The following initial conditions were used to perturb the airfoil at the start of simulation,

$$
\left\{\begin{array}{l}
h^{0}=0.001 \mathrm{~m} \\
\alpha^{0}=0.001 \mathrm{rad} \\
\dot{h}^{0}=0.0 \\
\dot{\alpha}^{0}=0.0
\end{array}\right.
$$

Initially, a steady solution is generated for the 2D typical section with prescribed initial displacements. From the steady solution, the aerodynamic loading is obtained, and the initial accelerations are computed. Then Eqs. (106) and (107) are used for the starting procedure.

Subsequently, the best coupling schemes are used to obtain the partitioned solution using an Euler CFD solver to verify the correctness of the coupling scheme, and compare it with the MN scheme. The flutter boundary is determined by V-g method, where the damping coefficients are extracted from system response by autoregressive moving average (ARMA) method [54].

Next, CFD-based solutions from schemes FH and MN are compared. The time responses for displacements and forces are shown in Fig. 19 and 20, where a solution using piston theory (labelled "pt") is also included for comparison. The responses of airfoil from scheme FH and MN agree well with each other. The time-accuracy and energy-accuracy of schemes FH and MN agree with theoretical prediction as well as numerical verifications using piston theory. Both schemes display second-order time-accuracy in displacements and forces. The FH scheme is fourth-order energyaccurate, while the MN scheme is third-order energy-accurate. The time accuracy of damping coefficient is one order lower than the order of energy accuracy. This illustrates the superiority of the FH scheme over the MN scheme in terms of accuracy and numerical stability. One can therefore speculate that the FH scheme may produce more accurate results for long-time-scale simulations than the MN scheme. 


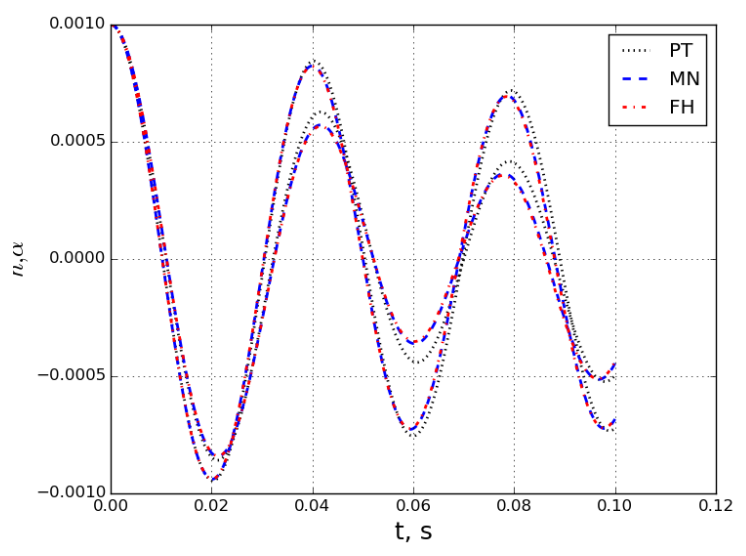

Figure 19. Comparison of displacements using schemes FH and MN.

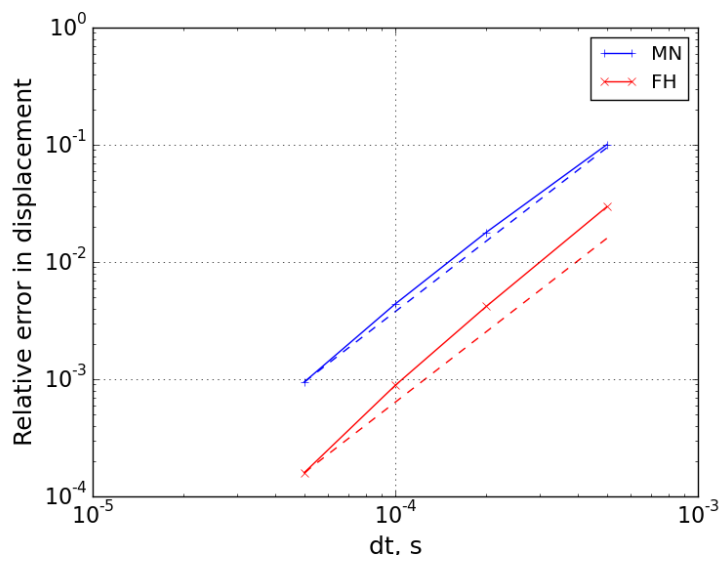

Figure 21. Relative error of displacements.

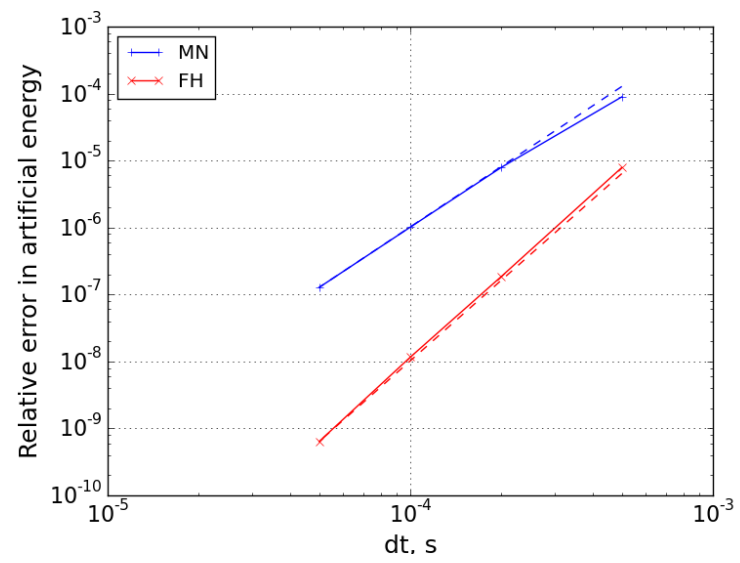

Figure 23. Relative error of artificial energy.

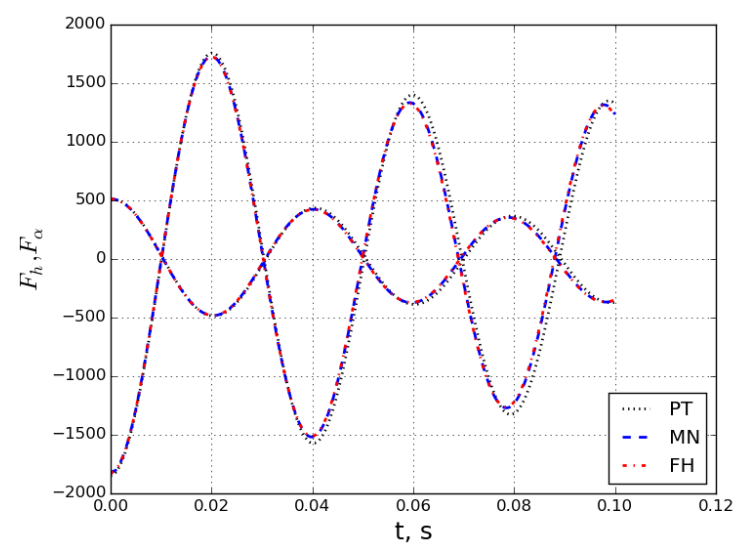

Figure 20. Comparison of forces using schemes FH and MN.

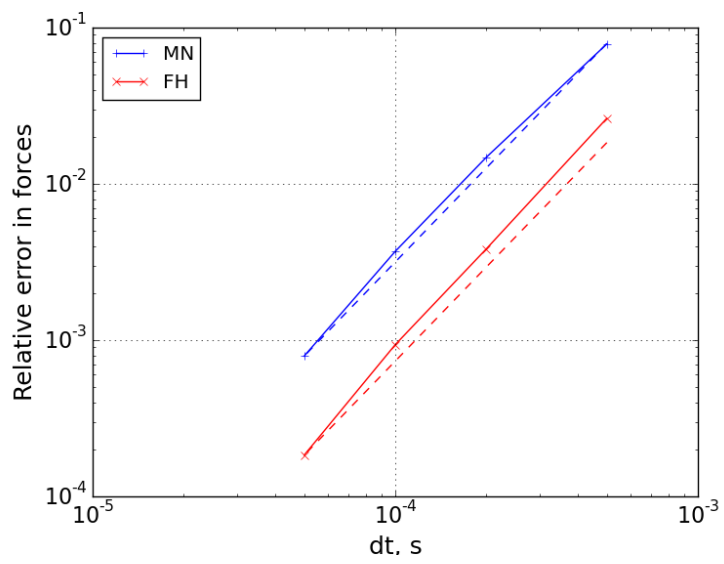

Figure 22. Relative error of forces.

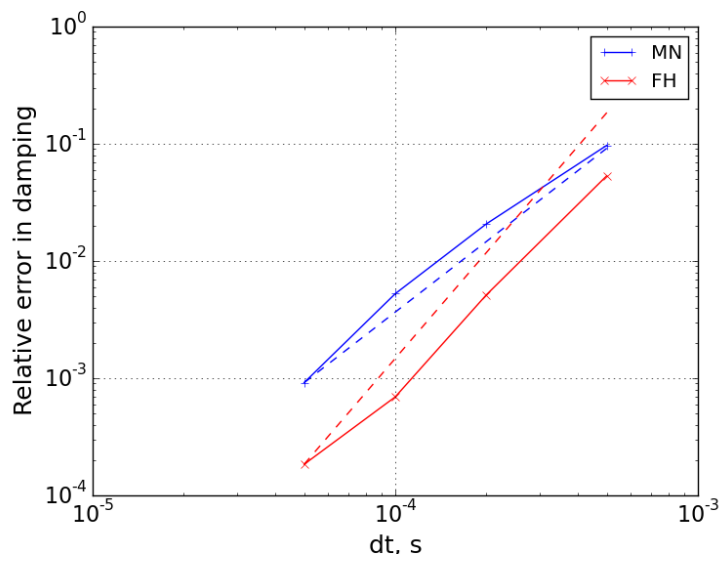

Figure 24. Relative error of damping coefficient. 


\section{Application to Panel Flutter}

\section{A. The computational models}

The configurations considered include a semi-infinite panel, i.e. a 2D panel, and a 3D panel with aspect ratio $a / b=1$. The computational model for 2D panel is shown in Fig. 25. The panel is discretized using 50 elements in streamwise direction with simply supported $\mathrm{BC}$ on its leading and trailing edges. The structural mesh is one-element wide in $y$ direction and symmetric $\mathrm{BC}$ are applied to the sides parallel to $x$ axis, i.e.

$$
v=0, \psi_{x}=0, \frac{\partial w}{\partial y}=0
$$

The fluid domain is discretized using structured mesh. The block over the panel is discretized into $128 \times 128$ cells in the $x z$ plane. The dimensions $l_{1}, l_{2}, l_{h}$ are adjusted to suit Mach numbers and Reynolds numbers for different cases. Particularly, the value of $l_{1}$ is for controlling the boundary layer thickness over the panel. For example, $l_{1}$ is increased when thicker boundary layer is desired over the panel. The computational model for 3D panel is shown in Fig. 26. The panel is discretized using a $16 \times 16$ mesh. The four sides of the panel are simply supported. In the fluid domain, the block over the panel is discretized into 64 cells both in streamwise and spanwise directions, and 128 cells in $z$ direction. In all the CFD meshes, the $y^{+}$values are kept under 0.5 for sufficient resolution of the boundary layer.

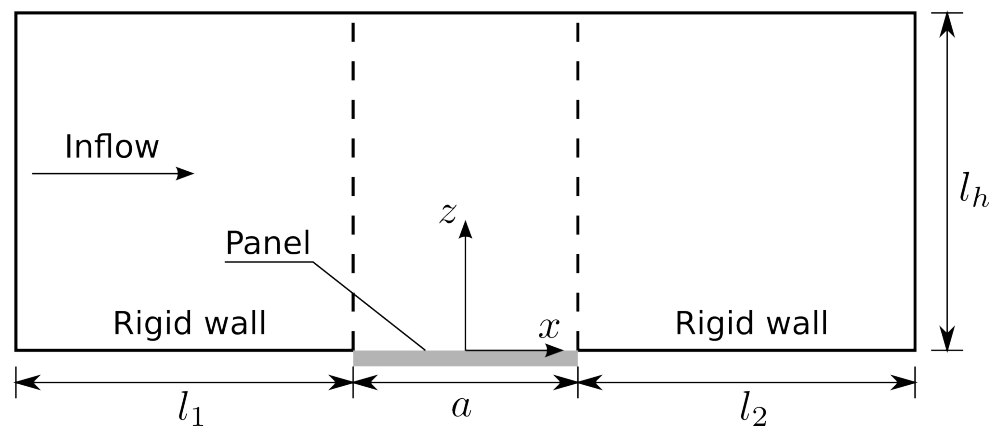

Figure 25. The computational model for 2D case.

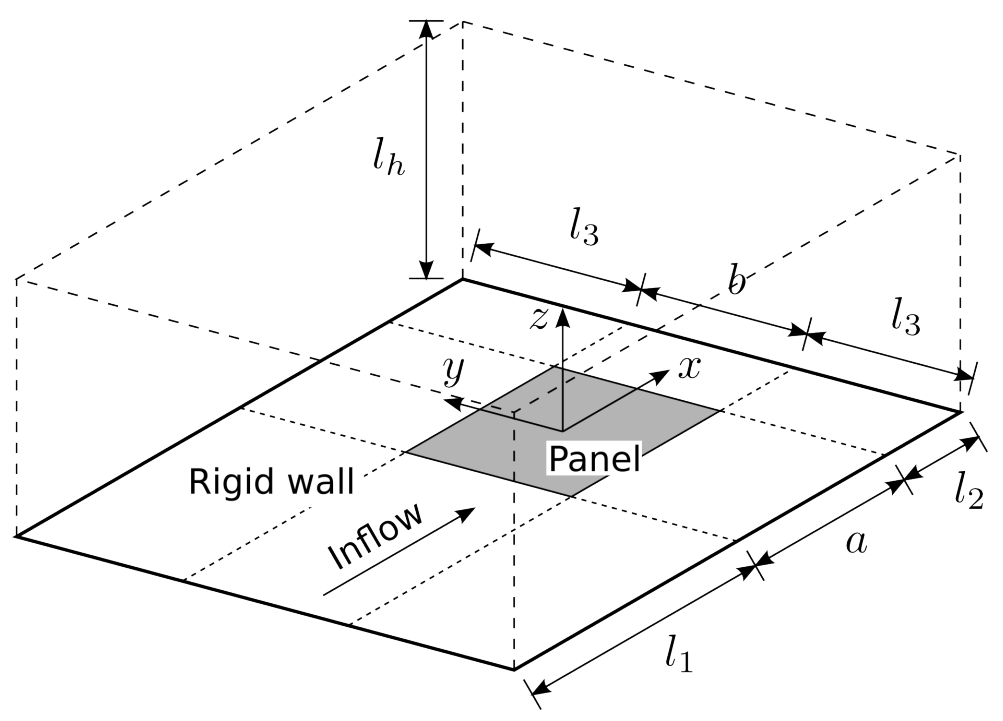

Figure 26. The computational model for 3D case.

In the CFD solution by an NS solver, due to the effect of boundary layer, the exterior static pressure on the panel is slightly larger than the freestream pressure $p_{\infty}$. To balance the effect of the pressure increase, the cavity pressure is set to be the average of the exterior pressures at the leading and trailing edges [8]. Typical cavity pressure ranges from $1.005 p_{\infty}-1.022 p_{\infty}$. 
The simulation is initialized as follows. Prior to the simulation, the panel is deformed using a small uniform load in $z$ direction. Then, a steady solution is generated for the deformed panel. From the steady solution, the aerodynamic loading is obtained. Finally, at the beginning of the simulation, Eqs. (106) and (107) are used for the starting procedure.

\section{B. Comparison with Gordnier's case}

This is a 2D case in supersonic flow treated in section 5.3.1 of Ref. [8]. The aspect ratio is $a / b=0$. The 3D case is the same as the $2 \mathrm{D}$ case, except that $a / b=1$, i.e. a square plate. The parameters for the two cases are listed in Table 7.

The results are shown in Fig. 27. The 2D results agree well with those obtained in Ref. [8]. The predicted flutter point is approximately 5\% lower and the LCO amplitude is higher. In the $3 \mathrm{D}$ case, the critical flutter parameter at bifurcation increases by a factor of 2. A similar trend was noted in Ref. [48]. The flutter parameter for $a / b=1$ is approximately 1.5 times of that in $2 \mathrm{D}$ case. The physical reason for the increased flutter parameter is due to the additional bending stiffness in spanwise direction of the panel, which reduces the oscillation amplitude of the panel.

Table 7. Case parameters

\begin{tabular}{cccccc}
\hline$M$ & $R e$ & $\mu$ & $h / a$ & $\nu$ & $\delta / a$ \\
\hline 1.2 & 100000 & 0.1 & 0.002 & 0.3 & 0.025 \\
\hline
\end{tabular}

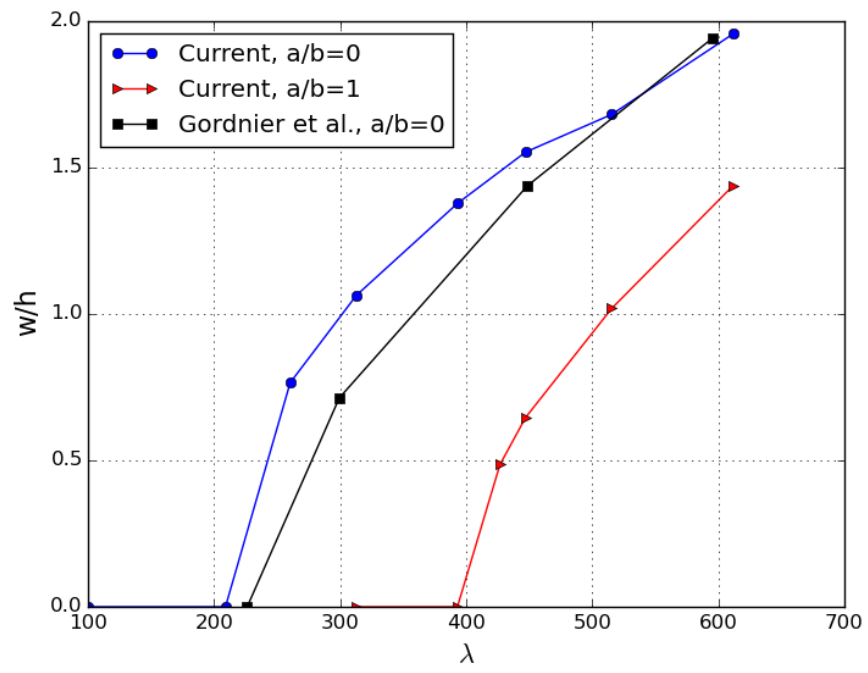

Figure 27. Effect of aspect ratio on flutter response.

\section{Effect of Reynolds number}

The effect of Reynolds number is explored using the $2 \mathrm{D}$ case to save computational time. In the modified $2 \mathrm{D}$ case, all parameters except $R e$ in Table 7 are retained. The Reynolds number is increased to $10^{6}$, which is a more practical value. In the CFD mesh, the length of the rigid wall region $l_{1}$ is increased to maintain $\delta / a=0.025$.

The results are shown in Fig. 28. Compared with the original 2D case, the critical flutter parameter is reduced by approximately $75 \%$. This may be due to the fact that at higher Reynolds number, the boundary layer growth is slower. As boundary layer over the panel becomes thinner, it reduces the value of critical flutter parameter [8]. 


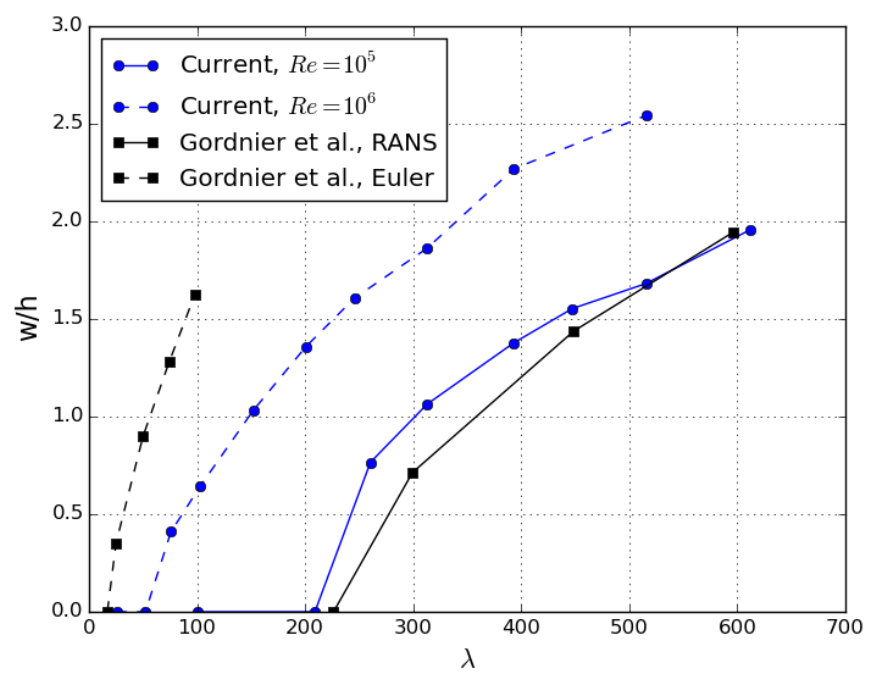

Figure 28. Effect of $R e$ on flutter response.

\section{A hypersonic case}

The parameters for this case are listed in Table 8. The plate material is Beryllium. The case is analyzed first using piston theory and then CFD. The results are shown in Fig. 29. The critical flutter parameter predicted using piston theory is approximately $M=5.3$. However, the critical value predicted using CFD is $M=5$, which is slightly lower. Furthermore, the LCO amplitude predicted by CFD-based solver is increased by a factor of 2 . The results suggest that piston theory can predict critical flutter parameter well, but it is not sufficient to characterize the post-flutter behavior of a panel.

Table 8. Case parameters

\begin{tabular}{cccccccc}
\hline$a$ & $b$ & $h$ & $M$ & $H$ & $\rho$ & $\nu$ & $E$ \\
\hline $1 \mathrm{~m}$ & $1 \mathrm{~m}$ & $0.0025 \mathrm{~m}$ & $4-8$ & $10 \mathrm{~km}$ & $1854 \mathrm{~kg} / \mathrm{m}^{3}$ & 0.1 & $290 \mathrm{GPa}$ \\
\hline
\end{tabular}

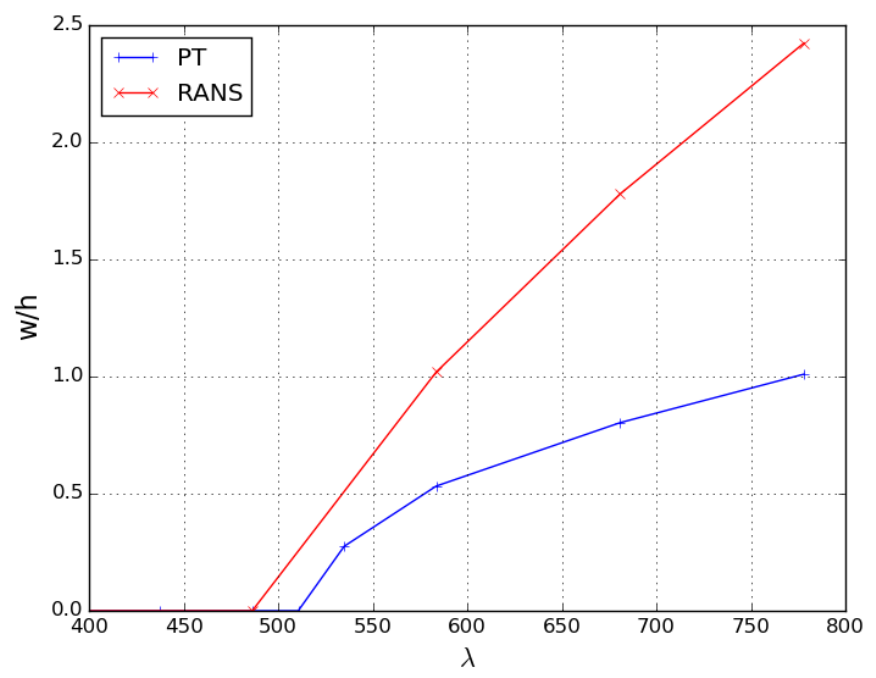

Figure 29. The LCO curve of a square panel in hypersonic flow. 


\section{Concluding Remarks}

An integrated aerothermoelastic analysis framework called HYPATE was developed. The framework is based on three components: a RANS unsteady aerodynamic solver, a FE-based structural solver, and a FE-based thermal solver. The framework couples the individual aerodynamic, structural, and thermal solvers using a partitioned scheme. The time accuracy of the framework is determined from the time accuracy of the loosely-coupled scheme and the so-called energy accuracy. The framework is extensively verified by comparison with a large number of representative test cases.

The framework is applied to the case of 2D and 3D panels by employing the aeroelastic portion of the framework in moderate supersonic flow. It is shown that the critical flutter parameter for the 3D case is twice that in the 2D case. The effect of the Reynolds number for the $2 \mathrm{D}$ case is explored by increasing it from $10^{5}$ to $10^{6}$, and the results show that the effect of the Reynolds number is important and cannot be ignored. A hypersonic 3D panel flutter case is also considered and compared to panels based on exact piston theory, and LCO amplitudes based on the CFD solution are approximately twice larger than piston theory, indicating that CFD is essential for generating reliable results.

\section{Acknowledgement}

The authors would like to acknowledge the help of Earl H. Dowell from Duke University and Abhijit Gogulapati from The Ohio State University.

\section{References}

[1] McNamara, J. J. and Friedmann, P. P., "Aeroelastic and Aerothermoelastic Analysis in Hypersonic Flow: Past, Present, and Future," AIAA journal, Vol. 49, No. 6, 2011, pp. 1089-1122.

[2] Miller, B. A. and McNamara, J. J., "Loosely Coupled Time-Marching of Fluid-Thermal-Structural Interactions with TimeAccurate CFD," AIAA Paper 2015-0686, 56th AIAA/ASCE/AHS/ASC Structures, Structural Dynamics, and Materials Conference, Kissimmee, Florida, Jan. 2015, pp. 1-16.

[3] Felippa, C. A., Park, K., and Farhat, C., "Partitioned Analysis of Coupled Mechanical Systems," Computer Methods in Applied Mechanics and Engineering, Vol. 190, No. 24, 2001, pp. 3247-3270.

[4] Farhat, C., Van der Zee, K. G., and Geuzaine, P., "Provably Second-Order Time-Accurate Loosely-Coupled Solution Algorithms for Transient Nonlinear Computational Aeroelasticity," Computer Methods in Applied Mechanics and Engineering, Vol. 195, No. 17, 2006, pp. 1973-2001.

[5] Culler, A. J. and McNamara, J. J., "Impact of Fluid-Thermal-Structural Coupling on Response Prediction of Hypersonic Skin Panels," AIAA Journal, Vol. 49, No. 11, 2011, pp. 2393-2406.

[6] Piperno, S. and Farhat, C., "Partitioned Procedures for the Transient Solution of Coupled Aeroelastic Problems - Part II: Energy Transfer Analysis and Three-Dimensional Applications," Comput. Methods in Applied Mechanics and Engineering, Vol. 190, No. 24, 2001, pp. 3147-3170.

[7] Mei, C., Abdel-Motagaly, K., and Chen, R., "Review of Nonlinear Panel Flutter at Supersonic and Hypersonic Speeds," Applied Mechanics Reviews, Vol. 52, No. 10, 1999, pp. 321-332.

[8] Gordnier, R. E. and Visbal, M. R., "Development of a Three-Dimensional Viscous Aeroelastic Solver for Nonlinear Panel Flutter," Journal of Fluids and Structures, Vol. 16, No. 4, 2002, pp. 497-527.

[9] Dugundji, J., "Theoretical Considerations of Panel Flutter at High Supersonic Mach Numbers." AIAA Journal, Vol. 4, No. 7, 1966, pp. 1257-1266.

[10] Friedmann, P. and Hanin, M., "Supersonic Non Linear Flutter of Orthotropic or Isotropic Panels with "arbitrary" Flow Direction," Israel Journal of Technology, Vol. 6, 1968, pp. 46-57.

[11] Falkiewicz, N. J., S. Cesnik, C. E., Crowell, A. R., and McNamara, J. J., "Reduced-Order Aerothermoelastic Framework for Hypersonic Vehicle Control Simulation," AIAA Journal, Vol. 49, No. 8, 2011, pp. 1625-1646.

[12] Lamorte, N. E., Uncertainty Propagation in Hypersonic Vehicle Aerothermoelastic Analysis, Ph.D. thesis, University of Michigan, 2013.

[13] Bapanapalli, S. K., Martinez, O. M., Gogu, C., Sankar, B. V., Haftka, R. T., and Blosser, M. L., "Analysis and Design of Corrugated-Core Sandwich Panels for Thermal Protection Systems of Space Vehicles," AIAA 2006-1942, 47th AIAA/ASME/ASCE/AHS/ASC Structures, Structural Dynamics, and Materials Conference, Newport, Rhode Island, 2006, pp. 1-18.

[14] Edwin van der Weide, Kalitzin, G., Schluter, J., and Alonso, J. J., "Unsteady Turbomachinery Computations Using Massively Parallel Platforms," AIAA Paper 2006-0421, 44th AIAA Aerospace Sciences Meeting and Exhibit, 2006, pp. 1-16.

[15] Donea, J., Giuliani, S., and Halleux, J., "An Arbitrary Lagrangian-Eulerian Finite Element Method for Transient Dynamic Fluid-Structure Interactions," Computer Methods in Applied Mechanics and Engineering, Vol. 33, No. 1, 1982 , pp. 689-723.

[16] Thomas, P. and Lombard, C., "Geometric Conservation Law and Its Application to Flow Computations on Moving Grids," AIAA Journal, Vol. 17, No. 10, 1979, pp. 1030-1037.

[17] Morton, S. A., Melville, R. B., and Visbal, M. R., "Accuracy and Coupling Issues of Aeroelastic Navier-Stokes Solutions on Deforming Meshes,” Journal of Aircraft, Vol. 35, No. 5, 1998, pp. 798-805. 
[18] Farhat, C., Geuzaine, P., and Grandmont, C., "The Discrete Geometric Conservation Law and the Nonlinear Stability of ALE Schemes for the Solution of Flow Problems on Moving Grids," Journal of Computational Physics, Vol. 174, No. 2, 2001, pp. 669-694.

[19] Bartels, R. E., "Mesh Strategies for Accurate Computation of Unsteady Spoiler and Aeroelastic Problems," Journal of Aircraft, Vol. 37, No. 3, 2000, pp. 521-525.

[20] Biedron, R. T. and Thomas, J. L., "Recent Enhancements to the FUN3D Flow Solver for Moving-Mesh Applications," AIAA Paper 2009-1360, 47th AIAA Aerospace Sciences Meeting Including The New Horizons Forum and Aerospace Exposition, Orlando, Florida, Jan. 2009, pp. 1-19.

[21] Geuzaine, P., Grandmont, C., and Farhat, C., "Design and Analysis of ALE Schemes With Provable Second-Order TimeAccuracy for Inviscid and Viscous Flow Simulations," Journal of Computational Physics, Vol. 191, No. 1, 2003 , pp. $206-227$.

[22] Koobus, B. and Farhat, C., "Second-Order Time-Accurate and Geometrically Conservative Implicit Schemes for Flow Computations on Unstructured Dynamic Meshes," Computer Methods in Applied Mechanics and Engineering, Vol. 170, No. 1, 1999, pp. 103-129.

[23] Reddy, J., Nonlinear Finite Element Analysis, Oxford University Press, New York, 2004, pp. 196-221.

[24] Park, M., Przekop, A., Phairoh, T., Huang, J.-K., and Mei, C., "Adaptive Control of Nonlinear Free Vibration of Shallow Shell Using Piezoelectric Actuators," AIAA Journal, Vol. 49, No. 3, 2011, pp. 472-488.

[25] Hughes, T. J., The Finite Element Method: Linear Static and Dynamic Finite Element Analysis, Prentice Hall, Englewood Cliffs, New Jersey, 1987.

[26] Bathe, K.-J. and Dvorkin, E. N., "A Formulation of General Shell Elements - the Use of Mixed Interpolation of Tensorial Components," International Journal for Numerical Methods in Engineering, Vol. 22, No. 3, 1986, pp. 697-722.

[27] Zienkiewicz, O. and Taylor, R., The Finite Element Method, Vol. 2: Solid and Fluid Mechanics/Dynamics and Non-linearity, McGraw-Hill, New York, 1991, pp. 216-243.

[28] Reddy, J. N., Mechanics of Laminated Composite Plates and Shells: Theory and Analysis, CRC Press, New York, 2004.

[29] Qatu, M. S., "Recent Research Advances in the Dynamic Behavior of Shells: 1989-2000, Part 1: Laminated Composite Shells," Applied Mechanics Reviews, Vol. 55, No. 4, 2002, pp. 325-350.

[30] Bathe, K.-J., Finite element procedures, Prentice Hall, Englewood Cliffs, New Jersey, 1996.

[31] Ashley, H. and Zartarian, G., "Piston Theory - a New Aerodynamic Tool for The Aeroelastician," Journal of the Aeronautical Sciences, Vol. 23, No. 12, 1956, pp. 1109-1118.

[32] Noor, A. K. and Burton, W. S., "Computational Models for High-Temperature Multilayered Composite Plates and Shells," Applied Mechanics Reviews, Vol. 45, No. 10, 1992, pp. 419-446.

[33] Noor, A. K. and Tenek, L. H., "Steady-State Nonlinear Heat Transfer in Multilayered Composite Panels," Journal of Engineering Mechanics, Vol. 118, No. 8, 1992, pp. 1661-1678.

[34] Argyris, J. and Tenek, L., "Recent Advances in Computational Thermostructural Analysis of Composite Plates and Shells With Strong Nonlinearities," Applied Mechanics Reviews, Vol. 50, No. 5, 1997, pp. 285-306.

[35] Bose, A. and Surana, K., "Piecewise Hierarchical P-Version Curved Shell Finite Element for Heat Conduction in Laminated Composites," Computers \& Structures, Vol. 49, No. 2, 1993, pp. 283-300.

[36] ABAQUS, ABAQUS 6.11: Theory Manual, Vol. 1, chap. 2, Dassault Systemes Simulia Corp., 2011, pp. 307-309.

[37] Muliana, A. H. and Kim, J. S., "A Two-Scale Homogenization Framework for Nonlinear Effective Thermal Conductivity of Laminated Composites," Acta Mechanica, Vol. 212, No. 3-4, 2010, pp. 319-347.

[38] Kaviany, M., Principles of Heat Transfer, chap. 1, John Wiley \& Sons, 2002, pp. 24-32.

[39] Lewis, R. W., Morgan, K., Thomas, H., and Seetharamu, K., The Finite Element Method in Heat Transfer Analysis, chap. 1, John Wiley \& Sons, 1996, pp. 13-28.

[40] Hughes, T. J., The finite element method: linear static and dynamic finite element analysis, chap. 8, Prentice Hall, Englewood Cliffs, New Jersey, 1987, pp. 459-478.

[41] Hughes, T. J., The finite element method: linear static and dynamic finite element analysis, chap. 4, Prentice Hall, Englewood Cliffs, New Jersey, 1987, pp. 217-231.

[42] Bendiksen, O. O., "A New Approach to Computational Aeroelasticity," AIAA paper 91-0939, Proceedings of AIAA/ASCE/AHS/ASC 32nd Structures, Structural Dynamics and Materials Conference, Baltimore, MD, April 1991, pp. 1712-1727.

[43] Miller, B. A., Crowell, A. R., and McNamara, J. J., "Loosely Coupled Time-Marching of Fluid-Thermal-Structural Interactions," AIAA 2013-1666, 54th AIAA/ASME/ASCE/AHS/ASC Structures, Structural Dynamics, and Materials Conference, Boston, Massachusetts, April 2013, pp. 1-17.

[44] Landon, R., "Compendium of Unsteady Aerodynamic Measurements," AGARD Report 702, 1982.

[45] McMullen, M. S., The Application of Non-linear Frequency Domain Methods to the Euler and Navier-Stokes Equations, Ph.D. thesis, Stanford, Stanford, CA, 2003.

[46] Pandya, M. J., Frink, N. T., and Noack, R. W., "Progress Toward Overset-Grid Moving Body Capability for USM3D Unstructured Flow Solver," AIAA Paper 2005-5118, 17th AIAA Computational Fluid Dynamics Conference, Toronto, Ontario Canada, June 2005, pp. 1-18.

[47] Nydick, I., Friedmann, P. P., and Zhong, X., "Hypersonic Panel Flutter Studies on Curved Panels," AIAA Paper 95-1485-CP, Proceedings of 34th AIAA/ASME/ASCE/AHS/ASC Structures, Structural Dynamics, and Materials Conference, New Orleans, LA, April 10-13 1995, pp. 2995-3011.

[48] Dowell, E. H., "Nonlinear Oscillations of a Fluttering Plate," AIAA Journal, Vol. 4, No. 7, 1966, pp. 1267-1275. 
[49] Gray, C. E. and Mei, C., "Large-Amplitude Finite Element Flutter Analysis of Composite Panels in Hypersonic Flow," AIAA Journal, Vol. 31, No. 6, 1993, pp. 1090-1099.

[50] Gogu, C., Bapanapalli, S. K., Haftka, R. T., and Sankar, B. V., "Comparison of Materials for an Integrated Thermal Protection System for Spacecraft Reentry,” Journal of Spacecraft and Rockets, Vol. 46, No. 3, 2009, pp. 501-513.

[51] Parthasarathy, T. A., Zawada, L. P., John, R., Cinibulk, M. K., Kerans, R. J., and Zelina, J., "Evaluation of Oxide-Oxide Composites in a Novel Combustor Wall Application," International Journal of Applied Ceramic Technology, Vol. 2, No. 2, 2005, pp. 122-132.

[52] David E. Myers, C. J. M. and Blosser, M. L., "Parametric Weight Comparison of Advanced Metallic, Ceramic Tile, and Ceramic Blanket Thermal Protection Systems," , National Aeronautics and Space Administration, 2000.

[53] Military handbook, MIL-HDBK-5H: Metallic Materials and Elements for Aerospace Vehicle Structures, chap. 7, U.S. Department of Defence, 1998, pp. 1-6.

[54] McNamara, J. J. and Friedmann, P. P., "Flutter Boundary Identification for Time-Domain Computational Aeroelasticity," AIAA Journal, Vol. 45, No. 7, 2007, pp. 1546-1555. 